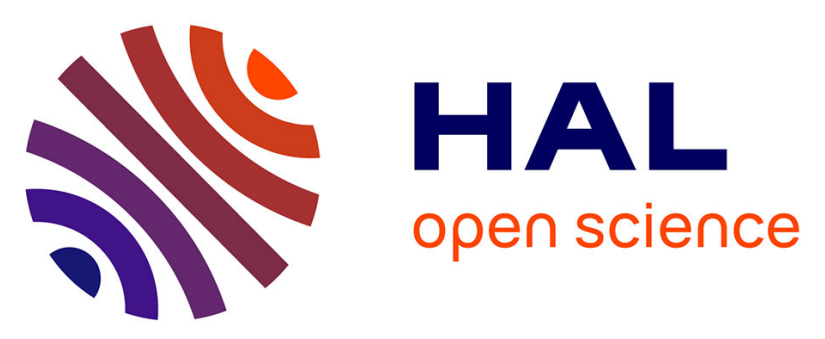

\title{
Indicateurs morphosédimentaires des variations de niveau lacustre dans les terrasses du lac de Van (Turquie)
}

Aurelien Christol, Catherine Kuzucuoğlu, Monique Fort, Damase Mouralis, Ali-Fuat Dogu, Ebru Akköprü, Daniel Brunstein, Michel Fontugne, Mustapha Karabiyikoglu, Stéphane Scaillet, et al.

\section{To cite this version:}

Aurelien Christol, Catherine Kuzucuoğlu, Monique Fort, Damase Mouralis, Ali-Fuat Dogu, et al.. Indicateurs morphosédimentaires des variations de niveau lacustre dans les terrasses du lac de Van (Turquie). Quaternaire, 2010, 21 (4), pp.443-458. 10.4000/quaternaire.5757 . hal-01333098

\section{HAL Id: hal-01333098 https://hal.science/hal-01333098}

Submitted on 19 Oct 2020

HAL is a multi-disciplinary open access archive for the deposit and dissemination of scientific research documents, whether they are published or not. The documents may come from teaching and research institutions in France or abroad, or from public or private research centers.
L'archive ouverte pluridisciplinaire HAL, est destinée au dépôt et à la diffusion de documents scientifiques de niveau recherche, publiés ou non, émanant des établissements d'enseignement et de recherche français ou étrangers, des laboratoires publics ou privés. 


\title{
INDICATEURS MORPHOSÉDIMENTAIRES DES VARIATIONS DE NIVEAU LACUSTRE DANS LES TERRASSES DU LAC DE VAN (TURQUIE)
}

\author{
Aurélien CHRISTOL ${ }^{1,2}$, Catherine KUZUCUOGLU ${ }^{2}$, Monique FORT ${ }^{1}$, \\ Damase MOURALIS ${ }^{3}$, Ali Fuat DOGU ${ }^{4}$, Ebru AKKÖPRÜ ${ }^{4}$, Daniel BRUNSTEIN ${ }^{2}$, \\ Michel FONTUGNE ${ }^{5}$, Mustafa KARABIYIKOGLU ${ }^{6}$, Stéphane SCAILLET ${ }^{5}$ \\ \& Halil ZORER ${ }^{4}$
}

\section{RÉSUMÉ}

Le lac de Van, dans les montagnes d'Anatolie orientale, ne possède pas d'exutoire naturel. Malgré son volume $\left(604 \mathrm{~km}^{3}\right)$, les amplitudes des variations de son niveau témoignent, depuis 1944, d'une sensibilité élevée aux fluctuations hydro-climatiques. Dans cette perspective, les dépôts hérités d'anciens hauts niveaux, conservés sous forme de terrasses côtières dans les parties aval anciennement immergées des affluents du lac, permettent d'identifier, de caractériser et de dater les changements de volume d'eau lacustre qu'ont enregistrés les sédiments qui sous-tendent ces terrasses. Plusieurs travaux récents sur les sédiments du fond du lac de Van ont mis en évidence leur intérêt exceptionnel pour la reconstitution des paléoclimats et permis de détailler l'évolution paléoenvironnementale du Tardiglaciaire et de l'Holocène. Cependant, les études des dépôts lacustres émergés restent rares. Ces dépôts font l'obje ici d'une étude géomorphologique de terrain couplée à des caractérisations sédimentologiques de faciès, des mesures altimétriques DGPS, des reconstitutions spatialisées et la mise en place des premiers jalons d'une chronostratigraphie basée sur des datations ${ }^{14} \mathrm{C}$ de dépôts organiques et coquilles, ${ }^{39} \mathrm{Ar} /{ }^{40} \mathrm{Ar}$ de tephras, et U-Th de travertins. Dans l'attente de datations OSL, nos résultats préliminaires permettent d'identifier au moins deux cycles de transgression, datés respectivement du début du dernier Glaciaire et d'une période couvrant le Dernier Maximum Glaciaire (DMG). Chaque cycle correspond à une phase de transgression rapide, suivie de variations intermédiaires d'amplitude plus ou moins forte, et se terminant par une longue phase de régression par paliers. La cause de ces variations et, notamment, la part qu'y a prise le climat est évidente pour le cycle datant du DMG, mais l'est moins pour celui marquant le début du Glaciaire dont les liens avec le volcanisme et la tectonique sont étroits. La forte instabilité de la paléogéographie est liée au volcanisme qui est à l'origine de barrages ayant entraîné la fermeture du bassin versant du lac.

Mots-clés : Lac de Van, terrasses, Pléistocène supérieur, sédimentologie, niveaux lacustres, Anatolie orientale.

\section{ABSTRACT}

MORPHOSEDIMENTARY EVIDENCES OF LAKE LEVEL VARIATIONS IN THE TERRACES OF LAKE VAN (TURKEY)

In the eastern Anatolian Mountains, the relatively high-magnitude variations exhibited by the level of Lake Van in recent years indicate, as the lake has no outlet, that this level can be considered as a sensitive indicator of climate if these variations respond to changes in water input/output. Until now, previous palaeoclimatic research has focused mainly on deep sediments in the lake, which provided an environmental sequence which is today a reference for the Late Glacial and the Holocene in the Middle East. In spite of many landforms and deposits around the lake pointing out to the occurrence of very high levels in the past, studies on the lacustrine sediments preserved in terrace records around the lake have remained quite rare since a first exhaustive overview realized in the 1970's. We here present the first results of a field work coupled with sedimentology studies (stratigraphy, facies analyses), DGPS elevation measurements, spatialized reconstructions. Chronostratigraphy takes advantage of the presence of interstratified tephras layers dated by ${ }^{39} \mathrm{Ar} /{ }^{40} \mathrm{Ar}$, of travertines dated by U-Th series, as well as of organic deposits and shells dated by ${ }^{14} \mathrm{C}$. Results evidence the occurrence of two major and rapid transgressions, the first one happening at the beginning of the Last Glacial, the second one in the millennia preceding the Last Glacial Maximum (LGM). Each identified lacustrine cycle started with a very rapid transgression followed by slower regressions with intermediate oscillations. Regressions started immediately after the highest stand was reached $(+80 \mathrm{~m}$ and $+55 \mathrm{~m})$. Regressions lasted long, with some stand-by during the descent, allowing erosion surfaces to truncate the surface of the emerging lake deposits. During Upper Pleistocene, tectonic and volcanic activities interfered with the climatic record of lake levels, both by deforming deposits locally (uplift, faulting) and by modifying the drainage area and river network of the lake basin (closure by damming, captures, isolation). Our preliminary results thus enlighten the complexity of the processes triggering the high magnitude transgressions of the Upper Pleistocene Van Lake, as well as the difficulty to establish correspondences between climatic changes and water volume changes as evidenced by past shoreline deposits preserved in the terrace sedimentary records.

Key-words : Lake Van, Terraces, Upper Pleistocene, sedimentology, lake levels, Eastern Anatolia.

\footnotetext{
${ }^{1}$ Université Paris7, UMR 8586 PRODIG, Equipe DYNMIRIS. UFR GHSS, Case 7001, Université Paris7-Denis Diderot, 2 place Jussieu,

75251 Paris Cedex 05. Courriel : aurelien.christol@wanadoo.fr

${ }^{2}$ Laboratoire de Géographie Physique, UMR 8591, CNRS-Université Paris1, 1 place Aristide Briand, 92195 Meudon cedex.

${ }^{3}$ Université de Rouen - CNRS, UMR 2795 IDEES, rue Lavoisier, 76821 Mont-Saint-Aignan.

${ }^{4}$ Université de Van, Département de Géographie, Cografya bölümü, Yüzüncü Yil Universitesi, 65080 Kampüs - VAN.

${ }^{5}$ Laboratoire des Sciences de 1'Environnement et du Climat, UMR 1572, CEA-CNRS, LSCE-Vallée, Bât. 12, avenue de la Terrasse,

91198 Gif-sur-Yvette Cedex.

${ }^{6}$ Université de Van, Département d'Anthropologie, Cografya bölümü, Yüzüncü Yil Universitesi, 65080 Kampüs - VAN.
} 


\section{1 - INTRODUCTION}

Le lac de Van est un site important pour la connaissance des variations climatiques et environnementales de Méditerranée orientale et du Moyen Orient. Les données pour l'Anatolie centrale et le Proche Orient font état de hauts niveaux lacustres au Dernier Maximum Glaciaire (DGM) (Kuzucuoğlu \& Roberts, 1997; Kuzucuoğlu et al., 1999; Bartov et al., 2002; Hazan et al., 2005), alors que les données paléoclimatiques régionales mettent en avant l'aridité de cette période (Robinson et al., 2006; Djamali et al., 2008). Cependant, les études paléoclimatiques qui concernent le Pléistocène supérieur dans cette région restent rares (Bar Mathews et al., 1997).

Dans le cadre d'une étude géologique régionale exhaustive (Degens \& Kurtmann, 1978), une séquence paléoclimatique a été établie à partir d'un carottage profond réalisé dans les sédiments varvés du lac en 1974 (van Zeist \& Woldring, 1978) qui concerne le Tardiglaciaire et l'Holocène. Renouvelés en 1990 (Landmann et al., 1996; Lemcke \& Sturm, 1997; Wick et al., 2003) et en 2004 (Litt et al., 2008), les carottages profonds du lac de Van livrent aujourd'hui des données paléoclimatiques à haute résolution qui remontent au DMG inclus (Heumann et al., 2008; Litt et al., 2008). Les reconstitutions établissent l'évolution de la végétation (van Zeist \& Woldring, 1978; Wick et al., 2003), du stress évaporitique mis en rapport avec des niveaux du lac (Landmann et al., 1996), de la température et de l'humidité (Lemcke \& Sturm, 1997). Les résultats des derniers carottages reconstituent l'évolution de la végétation depuis le DMG (Litt et al., 2008).

Les études menées sur les terrasses proposent des interprétations différentes. Schweizer (1975) identifie quatre niveaux de terrasses qu' $i l$ associe à quatre grandes phases climatiques (Tardiglaciaire, DMG, Würm, Würm-Riss). Valeton (1978) reconnaît une seule phase d'accumulation fluvio-lacustre suivie de plusieurs phases d'érosion et donne une date de $24 \mathrm{ka}$ BP pour le maximum de la transgression (+ $85 \mathrm{~m})$. Kempe et al. (2002) datent, eux, de 21-20 ka cal BP la terrasse de $+40 \mathrm{~m}$.

Afin de préciser les données paléoclimatiques contenues dans les sédiments du fond du lac, les dépôts hérités d'anciens hauts niveaux et préservés autour du lac sont précieux, car ils permettent de reconstituer le milieu de sédimentation et de positionner d'anciennes lignes de rivage; ils sont susceptibles de permettre des calculs de l'amplitude des variations du volume d'eau du lac et des bilans hydro-climatiques (Christol, 2005). Perchés au-dessus du réseau hydrographique actuel, les dépôts étudiés présentent des faciès d'origine lacustre et fluviolacustre. Leur étude montre qu'ils sont souvent tronqués par des surfaces d'érosion. Ces formations émergées autour du lac sont également marquées par l'impact d'événements tectoniques post-dépositionnels. Egalement, l'interstratification de tephras datables offre la possibilité de caler chronologiquement les niveaux du lac associés aux tephras avec des événements volcaniques.
Les résultats présentés ici ont été obtenus dans le cadre d'un programme de recherche pluridisciplinaire francoturc (ANOVAN: ECLIPSE II 2006-2007, TÜBITAK). Ils sont encore préliminaires puisque des datations OSL, ${ }^{39} \mathrm{Ar} /{ }^{40} \mathrm{Ar}$ et $\mathrm{K}-\mathrm{Ar}$ viendront en préciser la chronologie. Ces recherches sur le terrain ont eu lieu en 2005 et surtout en 2006 et 2007 (Kuzucuoğlu et al., 2007; Christol et al., 2008b; Kuzucuoğlu et al., 2008b). Les résultats présentés ici concernent deux des cinq vallées alimentant le lac: la Karasu et l'Engil, à l'est du lac. Il s'agit, dans un premier temps, de reconstituer et dater les variations du niveau du lac à partir des enregistrements sédimentaires dans les terrasses. Dans un second temps, il sera possible de discuter du rôle a) du forçage climatique sur les oscillations du niveau d'eau et du volume du lac, b) de l'impact des événements volcaniques ayant pu modifier la paléohydrographie du bassin versant.

\section{2 - PRÉSENTATION}

\section{1 - LOCALISATION ET PRÉSENTATION DU LAC DE VAN}

Le lac de Van est situé en Anatolie orientale à $42,4^{\circ}$ de longitude Est et $38,8^{\circ}$ de latitude Nord, au nord de la chaîne du Taurus oriental (fig. 1). Sa cote moyenne actuelle est de $1648 \mathrm{~m}$. La superficie du lac est de $3603 \mathrm{~km}^{2}$, soit un peu moins du tiers de celle du bassin versant $\left(11800 \mathrm{~km}^{2}\right)$. C'est un lac fermé, d'un volume de $614 \mathrm{~km}^{3}$ (quatrième rang mondial), et l'un des plus profonds avec une profondeur maximale de $460 \mathrm{~m}$ (Litt et al., 2008). Au sud du lac, les crêtes du massif de Bitlis dépassent les $3000 \mathrm{~m}$, culminant à $3479 \mathrm{~m}$. Au nord, le sommet du volcan Süphan atteint $4434 \mathrm{~m}$. Il est limité au sud par le bassin hydrographique du Tigre, à l'ouest par celui de l'Euphrate, au nord et à l'est par celui de l'Araxe.

Le lac de Van est le plus grand lac sodé du monde, avec un taux de salinité de $21,7 \%$ et un pH de 9,78. L'hyperalcalinité des eaux s'explique par des rejets fortement minéralisés liés à l'activité volcanique et hydrothermale, auxquels s'ajoutent les apports carbonatés par les rivières (Degens \& Kurtmann, 1978; Kipfer et al., 1994; Landmann et al., 1996; Lemcke \& Sturm, 1997; Kadioglu et al., 1997). Le fort taux de sodium de l'eau entraîne une absence de vie aquatique (et donc de matière organique dans les dépôts lacustres), sauf au débouché des rivières.

La largeur de la ceinture littorale, relativement plane, varie en fonction des vallées qui débouchent des chaînes montagneuses du sud-est, de l'est et du nord. Au sud-ouest, la limite du bassin versant est très proche du rivage, à un kilomètre seulement, car le drainage du massif de Bitlis est organisé vers le sud (bassin du Tigre) et non vers le lac. Au nord, le bassin versant est limité par l'alignement de quatre grands édifices volcaniques (fig. 1) qui isolent le lac du bassin de l'Euphrate (Nemrut et Süphan) et de l'Araxe (Meydan et Tendürek).

Le climat de la région du lac de Van est continental, caractérisé par de longs hivers froids, des étés chauds et de fortes amplitudes thermiques. A Van, les températures 


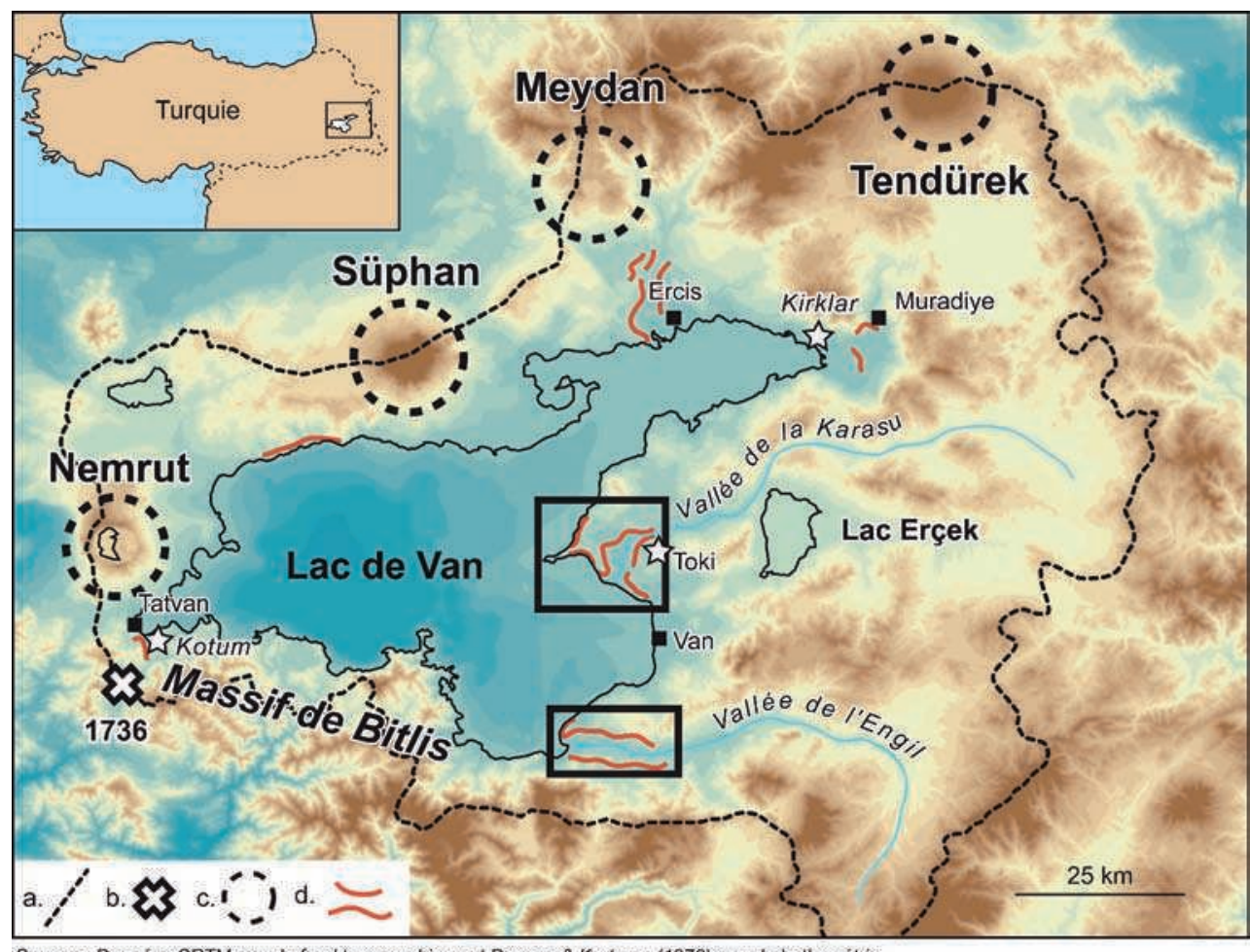

Fig. 1 : Présentation du bassin versant du lac de Van.

a. Limite du bassin versant; b. Seuil théorique de déversement des eaux; c. Principaux volcans et stratovolcans; d. Emplacement des principales terrasses autour du lac.

Fig. 1: The Lake Van basin: presentation.

a. Watershed limit; $b$. Threshold of the basin outflow; $c$. Main volcanoes and strato-volcanoes; d. Location of main terraces around the lake.

moyennes mensuelles pour la période 1951-1990 sont de $-3,1^{\circ} \mathrm{C}$ pour le mois de janvier et de $21,7^{\circ} \mathrm{C}$ pour le mois de juillet (Christol et al., 2008a). La sécheresse régionale est cependant modérée par l'effet orographique produit par le massif de Bitlis sur les masses d'air en provenance de l'ouest et par la recharge d'humidité due à l'évaporation des eaux de surface du lac. Ainsi, les fortes précipitations au sud-ouest du lac contrastent avec la faible pluviométrie des autres régions autour du lac. Cette configuration produit un fort gradient pluviométrique entre un secteur sud-ouest très arrosé (900 mm/an à Tatvan), rapidement décroissant vers la partie centrale $(300 / 400 \mathrm{~mm} /$ an, station de Van), et le secteur nord-oriental $(450 \mathrm{~mm} /$ an, station de Muradiye).

\section{2 - CONTEXTE GÉOLOGIQUE}

La géologie du bassin versant du lac de Van présente trois ensembles principaux (Degens \& Kurtman, 1978; carte géologique $1 / 500000^{\mathrm{e}}$ du MTA, 2002). Au sud, le massif de Bitlis (fig. 1) se compose essentiellement de roches métamorphiques paléozoïques. Les dépôts de la partie sud-orientale du bassin versant sont principalement des calcaires et des dépôts détritiques tertiaires marins (éocènes, oligocènes et miocènes) et continentaux (pliocènes). Ces calcaires présentent une karstification marquée de réseaux souterrains susceptibles d'influencer la dynamique hydrologique du lac et de ses affluents.

Au nord, un ensemble volcanique quaternaire composé de basaltes, andésites, rhyolites et pyroclastites recouvrent les sédiments tertiaires (Sengör \& Yilmaz, 1981; Yilmaz et al., 1998). Cet ensemble est principalement constitué de trois édifices alignés le long du littoral nord du lac. Les études menées sur le volcan Nemrut (Aydar et al., 2003) mais aussi sur les niveaux de tephra repérés dans les séquences de dépôts lacustres anciens et les couvertures de ponces repérées en surface des terrasses les plus hautes (Mouralis et al., 2007; Mouralis et al., 2010), confirment que l'activité volcanique s'est poursuivie tout au long du Pléistocène récent. Par ailleurs, le Nemrut (Aydar et al., 2003) et le Tendürek (1855 AD; www.volcano.si.edu et Jung et al., 1978) ont connu au moins une éruption historique et sont donc considérés comme actifs.

Par ailleurs, le bassin occupé par le lac est situé à une centaine de kilomètres à l'est du point de rencontre des failles coulissantes nord et sud-anatoliennes (Sengör et al., 2008). Celles-ci délimitent la plaque anatolienne, éjectée vers l'ouest du fait d'un mouvement rotatif de la plaque arabique. Cette collision inter-plaque engendre une tectonique active dans la région du lac de Van, continue depuis le Tertiaire. Elle est, par ailleurs, la cause du chevauchement du massif de Bitlis au-dessus de la dépression occupée par le lac (Sengör et al., 2008). Récemment, plusieurs failles de direction N-S et W-E ont rejoué dans le bassin versant du lac de Van. Certaines sont associées à un hydrothermalisme important (massif du Meydan, vallée de l'Engil). La configuration actuelle du réseau hydrographique (cours médian de la Karasu et de l'Engil) et de certains escarpements (ville de Van) confirment l'impact de la néotectonique sur l'organisation du relief. 


\section{3 - MÉTHODOLOGIE}

\section{1 - FACIÈS SÉDIMENTAIRES}

Les dépôts que l'on retrouve majoritairement sous les terrasses sont des sédiments lacustres laminés de granulométrie argilo-limoneuse qui correspondent à un milieu de lac profond de type bottomsets. D'autres dépôts lacustres présentent des faciès plus sableux et correspondent à des environnements proches du littoral (avant-côte). Parmi ceux-ci les sédiments les plus grossiers présentent parfois de très belles déformations d'origine sismique (sismites). Les dépôts proprement littoraux permettent de positionner directement un ancien haut niveau; ils sont alors sableux et graveleux, ou bien constitués de galets (cordons). Les dépôts les plus proximaux présentent des unités fossilifères à Dreissena et petits mollusques. Les terrasses sont parfois dessinées dans des ensembles deltaïques complexes de type Gilbert Delta avec leurs dépôts caractéristiques: bottomsets, foresets, topsets (Nemec \& Steels, 1988; Reading, 1996). Ces derniers montrent les mêmes faciès que des dépôts alluviaux (sables) que l'on trouve le plus souvent au sommet des terrasses. Les coupes dans les dépôts fluviatiles exposent des structures de stratification entrecroisée dans des sables et des graviers en alternance ou passage latéral avec des unités limoneuses qui correspondent à des milieux de plaine d'inondation. Ces alluvions permettent d'estimer les variations du profil des rivières en relation avec les variations du niveau du lac.

\section{2 - MORPHOLOGIES DE TERRASSE}

L'élaboration des terrasses correspond aux incisions et aux variations du profil des rivières, elles-mêmes déterminées par les changements du positionnement

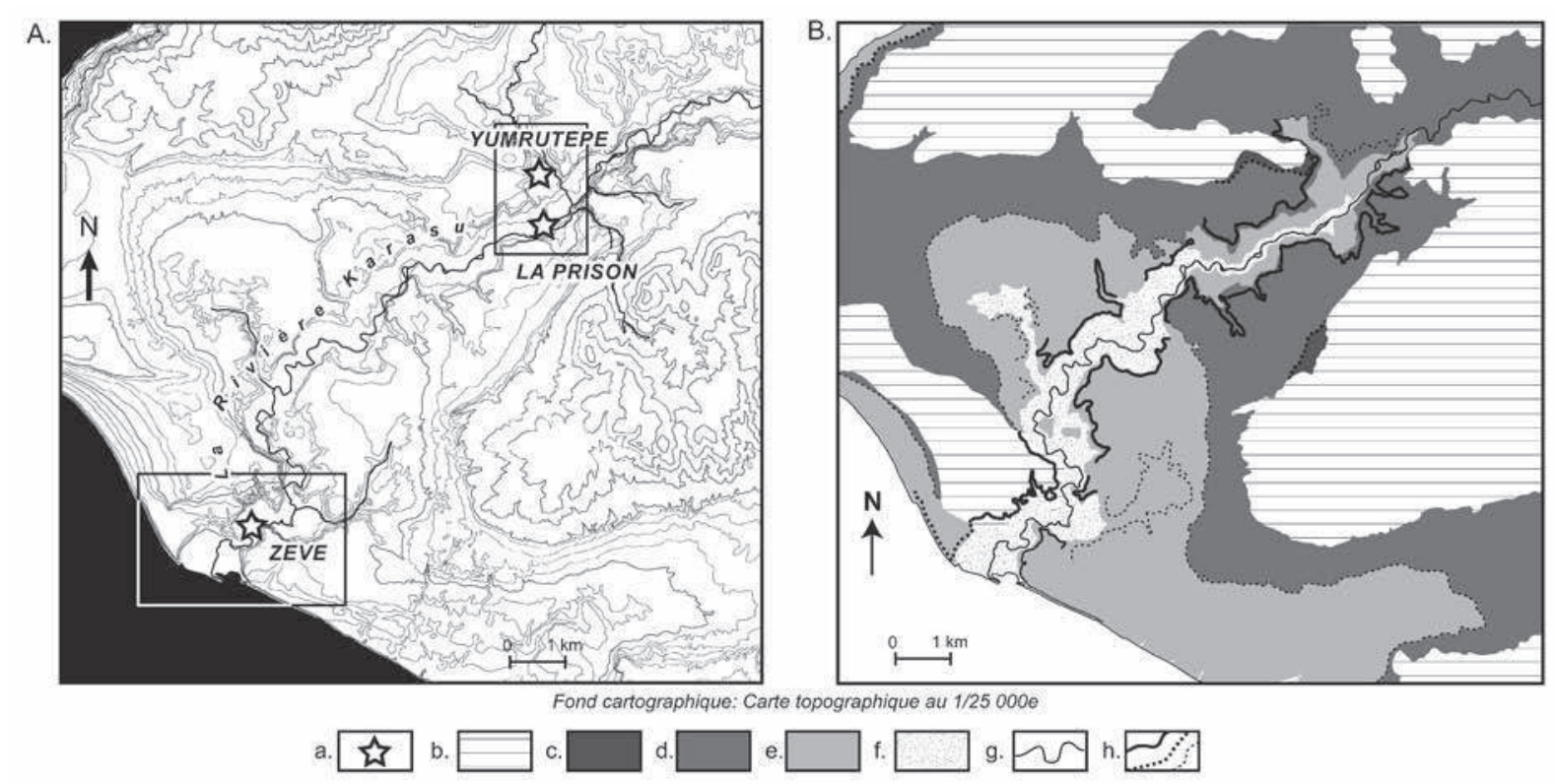

du niveau de base que constitue la surface du lac. Les morphologies des terrasses sont caractérisées par de vastes plans inclinés (d'amont en aval) limités par des talus bordiers. Ces deux éléments de la morphologie des terrasses répondent à des phases d'érosion de vitesses différentes qui ont affecté les accumulations sédimentaires dans lesquelles se dessinent les terrasses.

L'analyse morphosédimentaire de ces terrasses a nécessité un important travail de terrain afin d'identifier les morphologies utiles à la reconstitution des variations du niveau du lac. Ce travail s'est focalisé sur les parties aval des vallées de la Karasu et de l'Engil (fig. 2 et 3). La complexité de l'organisation spatiale des terrasses a nécessité de répertorier et de numéroter chaque terrasse ( $\mathrm{T} 1, \mathrm{~T} 2$, etc.) afin de procéder à des corrélations entre les deux vallées et de présenter un modèle d'interprétation morphologique et stratigraphique cohérent à l'échelle de chaque vallée (amont-aval), puis à celle des deux vallées considérées ensemble.

\section{3 - MESURES ALTIMÉTRIQUES ET MODÈLE NUMÉRIQUE DE TERRAIN (MNT)}

Les analyses morphosédimentaires ont été réalisées dans un cadre altimétrique précis afin de comparer les altitudes d'un site à l'autre malgré la contrainte de distance. Les relevés ont été effectués durant deux campagnes de mesures (2006 et 2007) avec un DGPS différentiel (équipement RTK-Thalès 6502), composé d'une station de base bi-fréquence et d'un appareil mobile (système géoïde EGM 96). Le niveau de référence du lac était à $1648 \mathrm{~m}$. Les 45 points levés sur chacune des deux vallées ont été référencés à partir d'une station primaire et de trois stations secondaires (tab. 1).

Des relevés altimétriques des contacts stratigraphiques ont également été effectués, afin de suivre

Fig. 2 : Présentation du secteur étudié à l'aval de la Karasu.

a. Sites principaux; b. Substrat géologique antéquaternaire; c. Dépôts du Cycle lacustre 1 potentiellement soulevés; d. Dépôts lacustres du Cycle 1; e. Dépôts du Cycle 2; f. Remplissage alluvial emboîté post-Cycle lacustre 2; g. Réseau hydrographique; h. Rebords plus ou moins marqués des terrasses. Fig. 2: Area studied in the downstream course of the Karasu River valley.

a. Main sites; b. Substratum; c. Lacustrine Cycle 1 deposits potentially uplifted; $d$. Lacustrine Cycle 1 deposits; e. Lacustrine Cycle 2 deposits; f. Alluvial fill post Cycle 2; g. Water network. h. Terraces scarp. 


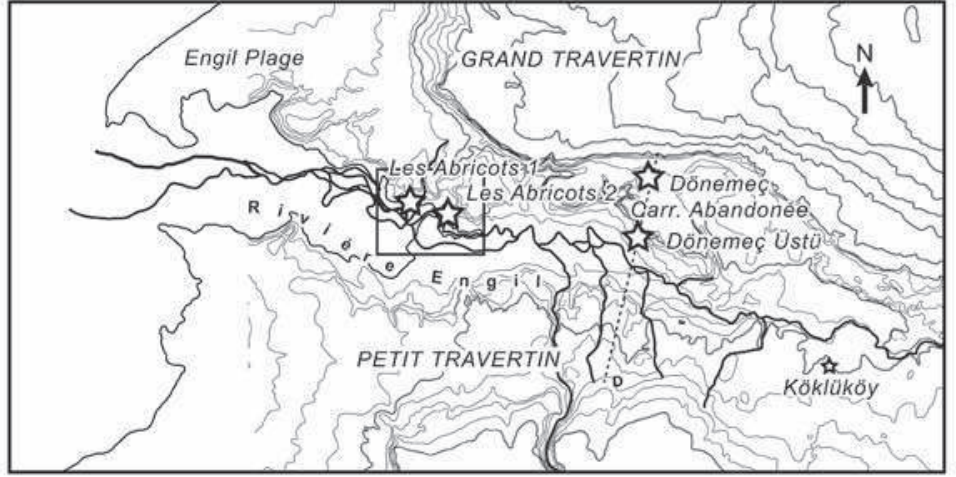

Fond cartographique: Carte topographique au 1/25 000e

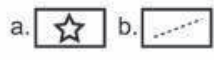

Fig. 3 : Présentation du secteur étudié à l'aval de l'Engil.

a. Sites principaux; b. Coupe de Dönemeç; c. Substrat; d. Dépôts du Cycle 1; e. Dépôts lacustres du Cycle 2 sous T3-T3' et dépôts du Cycle 2 sous T4 et T5; f. Remplissage alluvial post-Cycle 2 et lit majeur; g. Réseau hydrographique; h. Rebords de terrasses plus ou moins marqués.

Fig. 3: Area studied in the downstream course of the Engil River valley.

a. Main sites; b. Dönemeç section; c. Substratum; d. Cycle 1 deposits; e. Cycle 2 deposits associated with T3/T3'and T4/T5; f. Alluvial fill post-Cycle 2 and flood plain; g. River network. h. Terraces scarp.

\begin{tabular}{|c|c|c|c|c|c|c|c|c|}
\hline Nom Station & Description & & Type & Height & Longitude & Latitude & Préc. Horizontale & Préc. Verticale \\
\hline STUNIVERSI & & & Station principale & 1663,928 & $43^{\circ} 16^{\prime} 54.7707^{\prime \prime E}$ & $38^{\circ} 33^{\prime} 38.6104^{\prime \prime N}$ & 6.311 & 3,525 \\
\hline STENGIL1 & & & Station secondaire & 1685,142 & $43^{\circ} 11^{\prime} 25.0887^{\circ} \mathrm{E}$ & $38^{\circ} 20^{\prime} 58.9881 " \mathrm{~N}$ & 0,057 & 0,032 \\
\hline STYUM1 & & & Station secondaire & 1719,442 & $43^{\circ} 18^{\prime} 45.1924^{\prime \prime} \mathrm{E}$ & $38^{\circ} 40^{\prime} 28.1306^{\prime \prime N} \mathrm{~N}$ & 0 & 0 \\
\hline STZEVE1 & & & Station secondaire & 1686,336 & $43^{\circ} 14^{\prime} 06.7823^{\prime \prime} \mathrm{E}$ & $38^{\circ} 35^{\prime} 25.9160^{\prime \prime} \mathrm{N}$ & 0.036 & 0,02 \\
\hline Seuil & Seuil théorique de déversement des eaux & & Point levé & 1736,567 & $42^{\circ} 19^{\prime} 05.4138^{\prime \prime} E$ & $38^{\circ} 26^{\prime} 20.16269^{\prime \prime N}$ & 0 & 0,001 \\
\hline \multicolumn{9}{|c|}{ Numéro Point } \\
\hline & $1 \mathrm{~T} 1$ & Yumrutepe & Point Levé & 1745,493 & $43^{\circ} 18^{\prime} 30.1413^{\circ} \mathrm{E}$ & $38^{\circ} 40^{\prime} 23.3037 \mathrm{NN}$ & 0 & 0 \\
\hline & 2 T2 Yum & Yumrutepe & Point Levé & 1725,943 & $43^{\circ} 18^{\prime} 36.4686^{\circ} \mathrm{E}$ & $38^{\circ} 40^{\prime} 20.1713^{\prime \prime N}$ & 0.016 & 0.011 \\
\hline & $3 \mathrm{~T} 1$ surface à Yum 4 & Yumrutepe & Point Levé & 1740,355 & $43^{\circ} 18^{\prime} 24.2079^{\prime \prime} \mathrm{E}$ & $38^{\circ} 40^{\circ} 40.2253^{\prime \prime N}$ & 0 & 0 \\
\hline & 4 & Yumrutepe & Point Levé & 1712,983 & $43^{\circ} 18^{\prime} 41.0354^{\prime \prime} \mathrm{E}$ & $38^{\circ} 40^{\circ} 23.5712^{\prime \prime} \mathrm{N}$ & 0,027 & 0,007 \\
\hline & 5 Base Yum2 & Yumrutepe & Point Levé & 1694,969 & $43^{\circ} 18^{\prime} 46.9164^{\circ} \mathrm{E}$ & $38^{\circ} 40^{\prime} 24.7655^{\prime \prime N} \mathrm{~N}$ & 0,019 & 0,005 \\
\hline & 6 Incision dépôts de Yum 1 & Yumrutepe & Point Levé & 1699,031 & $43^{\circ} 18^{\prime} 38.5392^{\prime} \mathrm{E}$ & $38^{\circ} 39^{\prime} 55.0858^{\prime \prime N} \mathrm{~N}$ & 0,014 & 0,016 \\
\hline & 7 T3 à Yum1 & Yumrutepe & Point Levé & 1699,278 & $43^{\circ} 18^{\prime} 38.4885^{\circ} \mathrm{E}$ & $38^{\circ} 39^{\prime} 55.2950^{\prime \prime} \mathrm{N}$ & 0,015 & 0.013 \\
\hline & 8 Base dépôts de Yum 1 & Yumrutepe & Point Levé & 1676,685 & $43^{\circ} 18^{\prime} 44.2588^{\prime \prime} \mathrm{E}$ & $38^{\circ} 39^{\prime} 58.4866^{\prime \prime N}$ & 0.012 & 0.012 \\
\hline & 9 Niveau Karasu atuelle & Yumrutepe & Point Levé & 1672,732 & $43^{\circ} 18^{\prime} 25.3418^{\prime \prime} E$ & $38^{\circ} 39^{\prime} 28.4306^{\prime \prime N}$ & 0.014 & 0,011 \\
\hline & $10 \mathrm{~T} 2$ ' Sommet & Yumrutepe & Point Levé & 1702,348 & $43^{\circ} 18^{\prime} 17.5676^{\circ} \mathrm{E}$ & $38^{\circ} 39^{\prime} 19.3977^{\prime \prime N}$ & 0.215 & 0,251 \\
\hline & 11 T2' Sommet & Yumrutepe & Point Levé & 1701,966 & $43^{\circ} 18^{\prime} 17.5446^{\circ} \mathrm{E}$ & $38^{\circ} 39^{\prime} 19.3810^{\prime \prime N}$ & 0,018 & 0,015 \\
\hline & 12 T3 Sommet T3b & Zeve & Point Levé & 1688,466 & $43^{\circ} 14^{\prime} 14.8529^{\circ} \mathrm{E}$ & $38^{\circ} 35^{\prime} 28.9250^{\prime \prime N}$ & 0.011 & 0,006 \\
\hline & $13 \mathrm{~T} 4$ & Zeve & Point Levé & 1670,358 & $43^{\circ} 14^{\prime} 01.8316^{\circ} \mathrm{E}$ & $38^{\circ} 3540.8378^{\prime \prime N}$ & 0.039 & 0.033 \\
\hline & $14 \mathrm{~T}_{4}$ & Zeve & Point Levé & 1667,062 & $43^{\circ} 14^{\prime} 01.0880^{\circ} \mathrm{E}$ & $38^{\circ} 35^{\circ} 41.9017^{\prime \prime N}$ & 0.019 & 0,007 \\
\hline & 15 Contact alluvions-Dépóts lacustres & Zeve & Point Levé & 1665,457 & $43^{\circ} 14^{\prime} 06.0914^{\prime \prime} \mathrm{E}$ & $38^{\circ} 35^{\prime} 38.3433^{\prime \prime N} \mathrm{~N}$ & 0,032 & 0,012 \\
\hline & 16 Dépôts lacustres & Zeve & Point Levé & 1662,564 & $43^{\circ} 14^{\prime} 04.2565^{\circ} \mathrm{E}$ & $38^{\circ} 35^{\prime} 39.6675^{\prime \prime N}$ & 0,017 & 0,009 \\
\hline & 17 Dépôts lacustres & Zeve & Point Levé & 1654,131 & $43^{\circ} 14^{\prime} 04.7727^{\prime \prime E}$ & $38^{\circ} 35^{\prime} 39.9661 " \mathrm{~N}$ & 0,021 & 0,015 \\
\hline & 18 Depôts lacustres & Zeve & Point Levé & 1657,169 & $43^{\circ} 14^{\prime} 02.7793^{\prime \prime} \mathrm{E}$ & $38^{\circ} 35^{\circ} 41.3264^{\prime \prime N}$ & 0.016 & 0,007 \\
\hline & 19 Lit majeur de la Karasu & Zeve & Point Levé & 1648,042 & $43^{\circ} 14^{\circ} 03.4701^{\prime \prime} \mathrm{E}$ & $38^{\circ} 35^{\prime} 41.4151^{\prime \prime N}$ & 0,017 & 0,009 \\
\hline & 20 Niveau Karasu atuelle & Zeve & Point Levé & 1647,486 & $43^{\circ} 14^{\prime} 03.4674^{\circ} \mathrm{E}$ & $38^{\circ} 35^{\prime} 41.6911^{\prime \prime N} \mathrm{~N}$ & 0.023 & 0,008 \\
\hline & 21 T5 & Zeve & Point Levé & 1659,557 & $43^{\circ} 13^{\prime} 46,4549^{\prime \prime} E$ & $38^{\circ} 35^{\prime} 20.2283^{\prime \prime N}$ & 0,019 & 0,005 \\
\hline & $22 \mathrm{~T}^{\circ}$ & Zeve & Point Levé & 1661,156 & $43^{\circ} 13^{\prime} 49.1076^{\circ} \mathrm{E}$ & $38^{\circ} 35^{\prime} 20.3532^{\prime \prime N}$ & 0,008 & 0,005 \\
\hline & 23 T3 Sommet & Les Abricots1 & Point Levé & 1685,005 & $43^{\circ} 11^{\prime} 17.6799^{\circ} \mathrm{E}$ & $38^{\circ} 21^{\prime 2} 22.3005^{\prime \prime N}$ & 0,014 & 0,007 \\
\hline & 24 Base coupe les Abricots 1 & Les Abricots1. & Point Levé & 1660,622 & $43^{\circ} 11^{\prime} 23.7253^{\circ \mathrm{E}}$ & $38^{\circ} 20^{\prime} 57.3264^{\prime \prime N}$ & 0,008 & 0,007 \\
\hline & 25 Base unité à sismites & Les Abricots1 & Point Levé & 1671,641 & $43^{\circ} 11^{\prime} 24.1223^{\prime \prime} \mathrm{E}$ & $38^{\circ} 20^{\prime} 58.7780^{\prime \prime N}$ & 0.018 & 0.013 \\
\hline & 26 Incision latérale ouest & Les Abricots1 & Point Levé & 1675,964 & $43^{\circ} 11^{\prime 2} 23.6397^{\circ} \mathrm{E}$ & $38^{\circ} 20^{\prime} 59.0151^{\prime \prime N}$ & 0,042 & 0,018 \\
\hline & 27 Incision latérale est & Les Abricots1 & Point Levé & 1676,512 & $43^{\circ} 11^{\prime} 26.0504^{\circ} \mathrm{E}$ & $38^{\circ} 20^{\circ} 58.6588^{\prime \prime N} \mathrm{~N}$ & 0,019 & 0,01 \\
\hline & 28 Niveau plaine alluviale Engil & Les Abricots2 & Point Levé & 1650,846 & $43^{\circ} 11^{\prime} 22.8118^{\circ} \mathrm{E}$ & $38^{\circ} 20^{\prime} 43.1960^{\prime \prime N} \mathrm{~N}$ & 0,048 & 0,019 \\
\hline & 29 Niveau Engil acluelle & Les Abricots2 & Point Levé & 1649,728 & $43^{\circ} 11^{\prime} 22.4007^{\circ} E$ & $38^{\circ} 20^{\prime} 42.5794^{\prime \prime N}$ & 0.021 & 0,009 \\
\hline & 30 T6 & Les Abricots2 & Point Levé & 1658,755 & $43^{\circ} 11^{\prime} 47.1734^{\prime \prime} \mathrm{E}$ & $38^{\circ} 20^{\prime} 46.2373^{\prime \prime N} \mathrm{~N}$ & 0,052 & 0,014 \\
\hline & 31 Base alluvions conglomératiques & Les Abricots2 & Point Levé & 1655,423 & $43^{\circ} 11^{\prime} 46.9036^{\circ} \mathrm{E}$ & $38^{\circ} 20^{\prime} 46.8182^{\prime \prime N}$ & 0,017 & 0,004 \\
\hline & 32 Contact Plaine alluviale-dépôts T3 & Les Abricots2 & Point Levé & 1651,504 & $43^{\circ} 11^{\prime} 46.7146^{\circ} \mathrm{E}$ & $38^{\circ} 20^{\circ} 46.9312^{\prime \prime N}$ & 0,01 & 0,007 \\
\hline & 33 Sommet Alluvions conglomératiques & Les Abricots2 & Point Levé & 1659,051 & $43^{\circ} 11^{\prime} 48.8328^{\circ} \mathrm{E}$ & $38^{\circ} 20^{\prime} 47.1842^{\prime \prime N}$ & 0,007 & 0,006 \\
\hline & 34 Contact argiles et sables & Les Abricots2 & Point Levé & 1653,466 & $43^{\circ} 11^{\prime} 47.2270^{\circ} \mathrm{E}$ & $38^{\circ} 20^{\prime} 47.7285^{\prime \prime} \mathrm{N}$ & 0.016 & 0,006 \\
\hline & 35 Sommet Alluvions conglo. 2 & Les Abricots2 & Point Levé & 1658,839 & $43^{\circ} 11^{\prime} 47.6969^{\circ} \mathrm{E}$ & $38^{\circ} 20^{\prime} 49.8199^{\prime \prime N}$ & 0,019 & 0,009 \\
\hline & 36 Contact alluvions conglo-Dép.Lacustre & Les Abricots2 & Point Levé & 1660,633 & $43^{\circ} 10^{\prime} 46.7916^{\circ} \mathrm{E}$ & $38^{\circ} 21^{\prime} 09.5549^{\prime \prime N}$ & 0.019 & 0,004 \\
\hline & 37 T1' r.gauche & Dönemeç & Point Levé & 1699,427 & $43^{\circ} 14^{\prime} 06.9128^{\circ} \mathrm{E}$ & $38^{\circ} 19^{\prime} 48.9719^{\prime \prime N}$ & 0.028 & 0,026 \\
\hline & $38 \mathrm{~T} 2$ ' r.gauche & Dönemę̧ & Point Levé & 1690,323 & $43^{\circ} 14^{\prime} 07.3143^{\prime \prime} E$ & $38^{\circ} 19^{\prime} 54.8479^{\prime \prime N}$ & 0,015 & 0,009 \\
\hline & $39 \mathrm{~T}^{\prime}$ 'r gauche & Dönemeç & Point Levé & 1677,121 & $43^{\circ} 14^{\prime} 03.9607^{\prime \prime} \mathrm{E}$ & $38^{\circ} 20^{\prime} 02.7119^{\prime \prime N} \mathrm{~N}$ & 0,019 & 0,005 \\
\hline & $40 \mathrm{TS}^{\circ}$ & Dönemeç & Point Levé & 1668,401 & $43^{\circ} 13^{\prime} 49.0588^{\circ} \mathrm{E}$ & $38^{\circ} 20^{\prime} 13,4769^{\prime \prime} \mathrm{N}$ & 0,013 & 0,011 \\
\hline & 41 Sommet stromatolites $r$.droite & Donemeç & Point Levé & 1667,556 & $43^{\circ} 13^{\prime} 51.4357^{\prime \prime} \mathrm{E}$ & $38^{\circ} 20^{\prime} 18.9406^{\prime \prime N}$ & 0,018 & 0,011 \\
\hline & 42 Base stromatolites & Dőnemeç & Point Levé & 1662,063 & $43^{\circ} 13^{\prime} 49.3704^{\circ} \mathrm{E}$ & $38^{\circ} 20^{\prime} 19.44666^{\prime \prime N}$ & 0.008 & 0,007 \\
\hline & 43 Niveau Engil actuelle & Dönemeç & Point Levé & 1656,829 & $43^{\circ} 13^{\prime} 49.7889^{\prime \prime} \mathrm{E}$ & $38^{\circ} 20^{\prime} 18.3231^{\prime \prime} \mathrm{N}$ & 0,014 & 0,019 \\
\hline & 44 Sommet stromatolites r.droite & Dönemeç & Point Levé & 1673,005 & $43^{\circ} 13^{\prime} 55.0309^{\prime \prime} \mathrm{E}$ & $38^{\circ} 20^{\prime} 24.1360^{\prime \prime N}$ & 0.016 & 0.012 \\
\hline & $45 \mathrm{~T} 4 \mathrm{r}$. droite & Dönemeç & Point Levé & 1675,09 & $43^{\circ} 13^{\prime} 55.3151^{\prime} \mathrm{E}$ & $38^{\circ} 20^{\circ} 24.4925 " \mathrm{~N}$ & 0.02 & 0,007 \\
\hline & 46 T2 r.droite & Donemeç & Point Levé & 1686,45 & $43^{\circ} 13^{\prime} 47,1517^{\circ} \mathrm{E}$ & $38^{\circ} 20^{\prime} 32.9958^{\prime \prime} \mathrm{N}$ & 0,069 & 0,053 \\
\hline & $47 \mathrm{~T} 1$ & Dőnemeç & Point Levé & 1712,271 & $43^{\circ} 13^{\prime} 45.4829^{\circ} E$ & $38^{\circ} 20^{\prime} 38.8676^{\prime \prime N} \mathrm{~N}$ & 0,019 & 0,009 \\
\hline
\end{tabular}

Tab. 1 : Liste des points levés lors des campagnes DGPS 2006 et 2007 dans les vallées de l'Engil et de la Karasu.

Tab. 1: Index of the dgps points measured during the 2006 and 2007 DGPS campains in the Engil and Karasu Valleys.

une même unité d'une coupe ou d'un site à l'autre. Ces mesures étaient nécessaires à la réalisation d'une chronostratigraphie relative, étape indispensable avant l'échantillonnage pour l'élaboration d'une chronologie absolue.
A partir de l'altitude des anciens hauts niveaux du lac mesurée avec le DGPS et à l'aide d'un MNT (SRTM, résolution $90 \times 90 \mathrm{~m})$, on a pu reconstituer l'hypsométrie du bassin lacustre, ainsi que les volumes et surface occupés par le lac à différentes étapes de son évolution. 


\section{4 - DATATIONS}

Des datations ${ }^{14} \mathrm{C}$ ont été réalisées sur des niveaux organiques et des ensembles coquilliers provenant de dépôts littoraux. Des datations U-Th ont été réalisées sur des travertins, et des datations ${ }^{39} \mathrm{Ar} /{ }^{40} \mathrm{Ar}$ sur des plagioclases de retombées ponceuses (Mouralis et al., 2010). Toutes les dates présentées ont été réalisées au Laboratoire des Sciences du Climat et de l'Environnement (UMR 1572) où des datations sont encore en cours (notamment 10 dates ${ }^{39} \mathrm{Ar} /{ }^{40} \mathrm{Ar}$ ).

Mis à part des résultats sur coquilles $>30 \mathrm{ka}$ (en dehors, donc, des possibilités de la méthode), deux dates ${ }^{14} \mathrm{C}$ ont été obtenues dans des dépôts organiques de fin de transgression lacustre. La méthode est présentée dans Kuzucuoğlu et al. (2008b sous presse). Le tableau 2 présente ces dates en âge ${ }^{14} \mathrm{C}$ conventionnel et calibré avec le programme de Stuiver \& Reimer (1993). Pour l'instant nous ne disposons que de deux résultats ${ }^{39} \mathrm{Ar} /{ }^{40} \mathrm{Ar}$ : l'un concerne un échantillon prélevé à Topaktas (vallée de la Karasu; Est du lac) ; l'autre provient du secteur sudouest du lac (versant de la vallée de Kotum: fig. 1). La méthode de datation est décrite en détail dans Mouralis et al. (2010).

\section{4 - RÉSULTATS}

La rivière Karasu prend sa source à proximité de la crête frontière avec l'Iran. Son cours est relativement long $(140 \mathrm{~km})$. La rivière Engil $(120 \mathrm{~km})$ prend sa source dans le secteur oriental du massif de Bitlis (fig. 1). Les vallées que drainent ces deux rivières présentent une orientation E-O globalement rectiligne.

Dans le bassin de la Karasu, les formations géologiques sont principalement des argiles vertes d'âge tertiaire, des ensembles ophiolitiques et des calcaires. Les calcaires y affleurent souvent en éperons rocheux ou en collines tandis que les ophiolites forment des modelés plus doux. Le bassin de l'Engil est composé, au sud de roches métamorphiques, à l'est de calcaires et d'ophiolites, et à l'ouest de travertins quaternaires. Ces éléments structuraux du relief, la position et la nature du substrat, ont influencé la mise en place, la disposition et la conservation des systèmes de terrasses. Ainsi, la structure explique certaines différences morphologiques et sédimentologiques entre les terrasses conservées dans l'une et l'autre vallée.

Dans la Karasu, le secteur étudié comprend deux sections : a) à l'amont (Yumrutepe), la vallée est encaissée dans les terrasses et son cours y est sinueux; b) le secteur aval (Zeve) correspond à la plaine deltaïque actuelle, assez large mais contrainte par les affleurements d'un substrat plus résistant (calcaires) (fig. 2). Dans son cours aval, la rivière Engil coule dans une large plaine alluviale assez régulière (Dönemeç) qui débouche sur une vaste plaine deltaïque («Les Abricots») (fig. 3). Dans les deux vallées étudiées, le lit majeur actuel est incisé dans un système de terrasses séparées par des talus de hauteur décamétrique. L'expression topographique de ces terrasses, très repérable dans les paysages, possède une large extension spatiale (fig. 2B et 3B).

\section{1 - LA VALLÉE DE LA KARASU}

\subsection{1 - Faciès sédimentaires}

A Yumrutepe (amont, fig. 4), les dépôts de fond de lac sont visibles à la base des enregistrements (YUM1/YUM0) et dans les séquences associées à la terrasse supérieure (base de YUM2). L'unité de base, de $5 \mathrm{~m}$ d'épaisseur, montre des faciès argilo-limoneux laminés interrompus par plusieurs niveaux de ripple marks limono-sableux. Au dessus, une unité de 2,5 m d'épaisseur présente également des faciès à limons laminés; mais les lits plus épais indiquent un environnement proximal. Les coupes de Köprübasi, qui font face à Yumrutepe, sur l'autre rive, présentent également des faciès de séries lacustres principalement composées de limons laminés (Köprübasi 2) ou alternant avec des niveaux sableux (Köprübasi 1, fig. 4). Ces unités sableuses sont déformées par l'activité sismique (sismites).

Dans les coupes placées topographiquement au-dessus des précédentes, les enregistrements sédimentaires sont formés d'épais dépôts deltaïques de type Gilbert delta

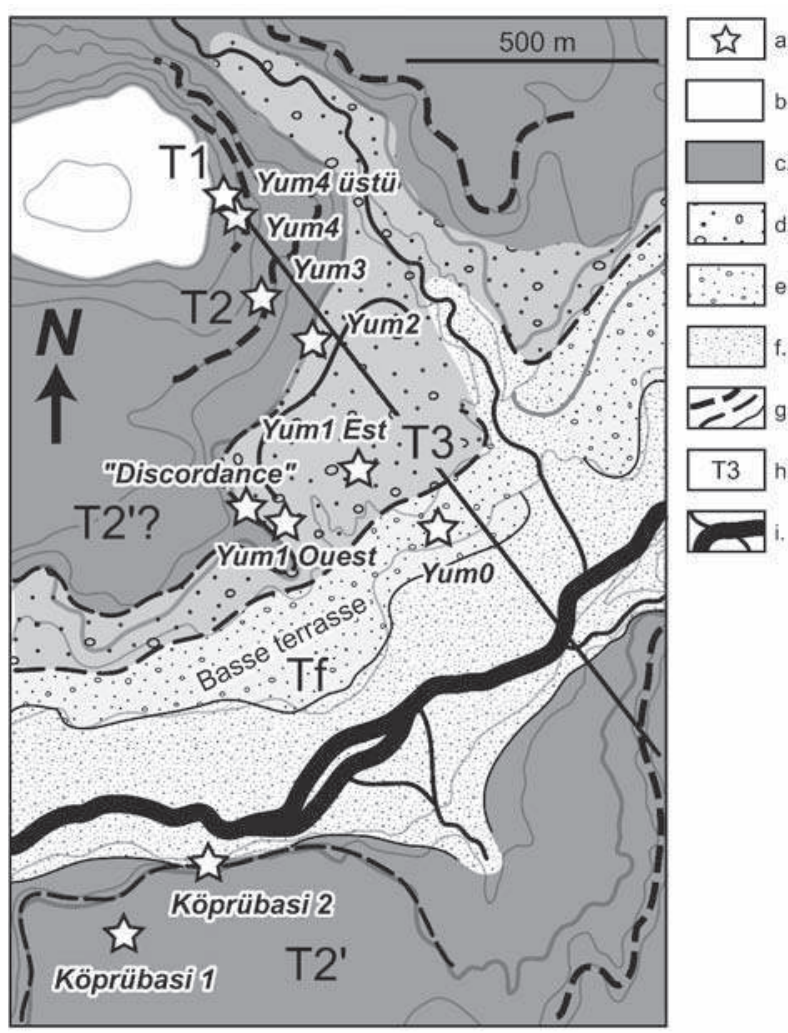

Fig. 4 : Carte géomorphologique du site de Yumrutepe (vallée de la Karasu).

a. Coupes ou ensemble de coupes (carrières) ; b. Substratum (calcaire) ; c. Dépôts du Cycle 1; d. Alluvions de la terrasse inférieure (associée au Cycle 2) ; e. Alluvions de la basse terrasse; f. Lit majeur; g. Rebords plus ou moins marqués des terrasses; h. Noms des terrasses; i. Réseau hydrographique.

Fig. 4: Geomorphological map of the Yumrutepe site (Karasu Valley). a. Section and group of sections (quarries); b. Substratum (limestones); c. Cycle 1 deposits; $d$. Alluvial terrace alluviums associated with Cycle 2; e. Low terrace alluvium; f. Flood plain; g. Terrace scarp; $h$. Names of the terraces; $i$. River network. 


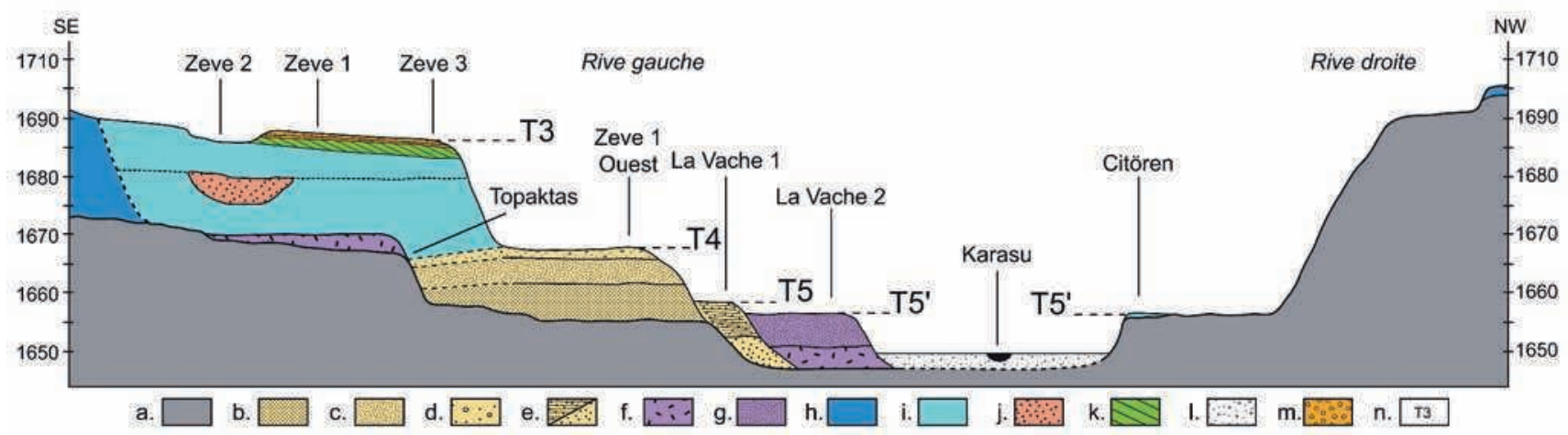

Fig. 5 : Coupe transversale de la Karasu à Zeve.

a. Substrat géologique; b. Sables fins alluviaux (pré-Cycle 1); c. Limons alluviaux (pré-Cycle 1); d. Alluvions grossières (pré-Cycle 1); e. Terrasse dans les alluvions pré-Cycle 1; f. Tephra en place (Topaktas) ou remaniée (Ravin de la Vache) ; g. Dépôts lacustres du début de la transgression du Cycle 2 ; h. Dépôts lacustres transgressifs du Cycle 1; i. Dépôts transgressifs du Cycle 2; j. Alluvions de régression intermédiaire dans le Cycle 2; k. Foresets de fin de transgression; 1 . Remplissage alluvial post-Cycle 2; $\mathrm{m}$. Colluvions; $\mathrm{n}$. Surfaces des terrasses.

Fig. 5: Cross section of the Karasu Valley at Zeve site.

a. Substratum; b. Alluvial sand deposits (pre-Cycle 1); c. Silty alluvium (pre-Cycle 1); d. Coarse alluvium (pre-Cycle 1); e. Pre-Cycle 1 alluvial terrace; $f$. In situ (Topaktas) or reworked (Ravin de la Vache) tephra; g. Cycle 2 transgression initial deposits; $h$. Cycle 1 transgressive deposits; $i$. Cycle 2 transgressive deposits; $j$. Regression alluvium during Cycle 2; $k$. Foresets related to terminal transgression; $l$. Post-Cycle 2 alluvial fill; $m$. Colluvium. n. Terrace surfaces.

(haut de YUM2). A Yumrutepe les plus grands foresets donnent des accumulations de $9 \mathrm{~m}$ (YUM2) et de 5 m (YUM3) soulignant la profondeur du lac. Dans les dépôts sommitaux de Yumrutepe, le passage des foresets aux topsets a permis de reconstituer la ligne de rivage au niveau de YUM2 et de YUM3. Au sommet de l'accumulation, le passage latéral d'une unité sableuse fine d'arrière plage à une unité plus grossière d'avant plage (YUM4) traduit par ailleurs un milieu de transition à l'endroit d'un ancien rivage.

Les carrières les plus basses (YUM 0) permettent d'établir qu'une basse terrasse et une très basse terrasse sont toutes deux d'origine alluviale (fig. 4). Les faciès y sont majoritairement sableux et grossiers (YUM1/YUM0) avec des structures lenticulaires en stratification entrecroisée, des chenaux et des unités massives de charges de fond traduisant une prédominance de l'aggradation dans un contexte d'ennoyage progressif (montée du niveau lacustre).

\subsection{2 - Identification des terrasses}

Grâce à ses carrières, le site de Yumrutepe permet l'étude des dépôts formant les plus hautes terrasses observées dans la vallée de la Karasu. Au moins trois niveaux de terrasses y ont été repérés, notamment en rive droite de la rivière (fig. 4). La terrasse supérieure (T1) se raccorde au versant calcaire par une surface d'érosion recoupant des dépôts de nature distincte. Cette surface est un plan incliné de 1745 à $1740 \mathrm{~m}$, peu étendu, qui se termine à l'aval par un talus de plusieurs mètres d'amplitude. Au pied de ce talus se développe une seconde terrasse (T2), nettement plus étendue et inclinée de 1725 à $1720 \mathrm{~m}$. Le talus qui sépare $\mathrm{T} 1$ et $\mathrm{T} 2$ suggère une première phase d'incision. En rive gauche (site de Köprübasi), il est possible de distinguer une troisième terrasse (T2') culmi- nant à $1702 \mathrm{~m}$ (fig. 4). En rive droite, une surface T2" à $1699 \mathrm{~m}$ se distingue de toutes les autres car elle correspond au toit de séries alluviales (YUM1) non tronquées qui se termine par des limons fluviatiles d'inondation (fig. 5). Dans le fond de la vallée, en rive droite, une basse terrasse fluviatile $(\mathrm{Tf})$ culmine à $+15 \mathrm{~m}$ au-dessus de la rivière (1687 m). Du fait de l'encaissement des méandres actuels aux sites de Yumrutepe-Köprübasi, le lit majeur de la rivière $(1672 \mathrm{~m})$ est dissymétrique avec une rive droite beaucoup plus large où sont préservés les dépôts fluviatiles absents en rive gauche.

Au site de Zeve proche de l'embouchure de la Karasu, la configuration des terrasses fait place à un autre système, caractérisé par un niveau principal très étendu (T3 - fig. 5), qui correspond à une surface d'accumulation lacustre dont l'altitude décroît progressivement vers l'aval jusqu'à $1688 \mathrm{~m}$. A Zeve, cette terrasse T3 correspond au toit d'accumulations sédimentaires non tronquées (à l'inverse de T2 à Yumrutepe qui recoupe des accumulations plus anciennes). T3 à Zeve domine les paysages de la rive gauche, tandis qu'en rive droite un niveau topographique supérieur peu développé est parsemé de gros galets vers $1695 \mathrm{~m}$ d'altitude. Ce niveau de rive droite, qui domine la terrasse T3 largement plus étendue, recoupe principalement le substrat. Sous T3, deux terrasses T4 et T5 sont respectivement positionnées à $1670 \mathrm{~m}$ et $1661 / 1659 \mathrm{~m}$ (fig. 5). Cellesci tronquent indifféremment a) la base de séries lacustres anciennes, b) des dépôts alluviaux antérieurs aux séries lacustres, c) le substrat déformé (couches d'argiles et de calcaires tertiaires à pendage vertical). Ainsi, dans le fond de la vallée en rive droite (Çitören, fig. 5), une très basse terrasse d'érosion ( $+5 \mathrm{~m}$ au-dessus de la rivière) recoupe des bottomsets lacustres conservés sur un mètre d'épaisseur, qui recouvrent en discordance horizontale un substrat tectonisé (calcaires tertiaires). 


\subsection{3 - Interprétations morpho-stratigraphiques}

Les unités stratigraphiques sont associées à des phases de transgression, de régression, de stabilité du niveau d'eau, d'incision et d'accumulation. La première transgression commence avec les unités laminées (I, Cycle 1a) sous les dépôts de YUM1/YUM0 visibles principalement à cet endroit (1677 m) (fig. 6). Les unités à la base de la coupe du site de Köprübasi 2 (IIa et IIb) jalonnent une deuxième phase de transgression (II, Cycle 1b) sans niveaux intermédiaires de régression. A Yumrutepe, les séquences lacustres sont continues avec à la base une unité de sédiments lacustres (IIc) surmontée par une unité deltaïque ancienne (foresets IId) recouverte par une seconde unité lacustre (IIe). La succession se poursuit par une seconde unité deltaïque à foresets (IIf). Ces unités témoignent de l'importance de la première transgression (Cycle 1) avec des variations dans la dynamique sédimentaire côtière liées à la plus ou moins grande proximité des versants ou de l'embouchure de la rivière (cône deltaïque). D'anciens niveaux du lac sont repérables à $1713 \mathrm{~m}$ (YUM2a) et 1725 m (YUM3). A YUM4 s'effectue la transition entre les unités transgressives et les unités finales proprement littorales (IIg) qui permettent de fixer la ligne de rivage à $1740 \mathrm{~m}$ d'altitude. Ces dépôts côtiers très élevés ont été soulevés par le jeu d'une faille normale dont le rejet cumulé atteint au moins $4 \mathrm{~m}$, amplitude calculée à partir du décalage entre des lits identiques repérés à YUM4 Üstü et à YUM4.

Les dépôts inférieurs de Yumrutepe (YUM1) sont emboîtés dans les séries lacustres (I et II) qui les dominent (fig. 6). Ce contact montre une incision recoupant des bottomsets équivalents à ceux de YUM2 (IIc). L'incision est scellée par des dépôts alluviaux d'inondation qui terminent la séquence fluviatile gravelo-sableuse développée plus bas sur 20 mètres d'épaisseur (YUM1).

Plus bas et à $+15 \mathrm{~m}$ d'altitude relative, une terrasse fluviatile (YUM0) formée dans la vallée est composée de dépôts alluviaux emboîtés dans les dépôts fluviatiles de YUM1. Il s'agit de la formation la plus récente du site de Yumrutepe (fig. 6).

A Zeve, la série sédimentaire transgressive (III), différente de celles de Yumrutepe-Köprübasi du point de vue stratigraphique, compose l'essentiel des dépôts de la terrasse principale (T3). Les accumulations à Zeve 3 atteignent une vingtaine de mètres au total (fig. 5 et 7 ) et correspondent à un second cycle lacustre (Cycle 2).

Le site de Zeve se caractérise par l'affleurement de formations fluviatiles antérieures à la séquence lacustre III. Le substrat affleure à Zeve 1 ouest mais les unités qui le recouvrent sont alluviales (Sub 2, 3 et 4). La coupe du ravin de la Vache 1 montre une formation Sub 5 alluviale emboitée dans les unités de Zeve 1 ouest. Une centaine de mètres en aval (Ravin de la Vache 2), les premières unités transgressives de Zeve 1 reposent sur un tephra remanié (Sub 6) qui fossilise des paléo-topographies. En amont de Zeve 1, ce tephra affleure in situ à
Topaktas où il est recouvert aussi par les dépôts de base de la série lacustre III (fig. 7).

Dans la coupe de Zeve 3 localisée entre Zeve 1 et Topaktas (fig. 7), la séquence de Zeve 1 se poursuit avec une série lacustre continue (IIIb-IIId) qui se termine par une unité à foresets (IIIc) formés d'apports fluviatiles déposés à proximité d'une ancienne ligne de rivage (IIId à $1685 \mathrm{~m})$.

Au nord du site de Zeve 3, dans le vallon de Zeve 2, la série lacustre est interrompue par une phase alluviale (absente à Zeve 3) recouverte par des dépôts lacustres de l'unité III (fig. 5). Cette configuration morphosédimentaire témoigne vraisemblablement d'un ancien tracé de la Karasu pendant le cycle 2 (fig. 7).

Les régressions qui ont suivi la transgression de Yumrutepe-Köprübasi (Cycle 1) ou celle de Zeve (Cycle 2) sont à l'origine de l'élaboration des terrasses. Le niveau atteint lors de la régression postérieure à la transgression de Zeve, a été plus bas que l'actuel. L'ampleur de la baisse de niveau de base a été telle que le réseau hydrographique s'est incisé dans des formations très anciennes (Sub 2, 3, 4 et 5-fig. 7) antérieures aux dépôts lacustres, ou plus récentes que celles des transgressions des Cycles 1 et 2 (IIIa et Sub 6).

\section{2 - LA VALLÉE DE L'ENGIL}

\subsection{1 - Faciès sédimentaires}

A l'amont de l'Engil (site de Dönemeç: fig. 3), les sédiments lacustres affleurant dans les talus correspondent à une accumulation ininterrompue de dépôts de fond de lac depuis $1670 \mathrm{~m}$ jusqu'à $1712 \mathrm{~m}$ (fig. 8). A «DönemeçCarrière abandonnée» affleure la plus haute série lacustre de la vallée. La surface d'érosion qui en tronque le sommet est le point culminant des dépôts. Dans une coupe située à hauteur équivalente à la précédente (Dönemeç Üstï), les dépôts sont essentiellement sableux, bien laminés ou massifs, avec des couches présentant plusieurs niveaux de petites sismites (fig. 8). La présence, à la même altitude, de dépôts de nature différente (bottomsets argileux dans la coupe haute, lits sableux et silteux dans la coupe basse) témoigne de la succession, ici, de deux environnements différents. De plus, la séquence de la «Carrière abandonnée» est interrompue (il manque les unités supérieures), contrairement à celle de Dönemeç Üstü qui est complète. Ces éléments combinés ne peuvent s'expliquer que par un emboîtement de la série «basse » (Cycle 1b) dans des dépôts plus anciens et de lac très profond (Cycle 1a) conservés à «Dönemeç-Carrière abandonnée».

En rive gauche, au site de Köklüköy $(1700 \mathrm{~m})$, les dépôts en bas de coupe sont limoneux et laminés, témoignant de la rapidité de la transgression ( $\mathrm{Ib}$ ?) tandis que la partie supérieure de la coupe correspond à des dépôts plus sableux indiquant un lac de faible profondeur et peut-être déjà le début de la régression. Comme à Dönemeç Üstü, ces niveaux sableux sont affectés de sismites.

Au pied de la grande coupe de Dönemeç Üstü, des sables contenant des passées grossières $(c f$. base de la 


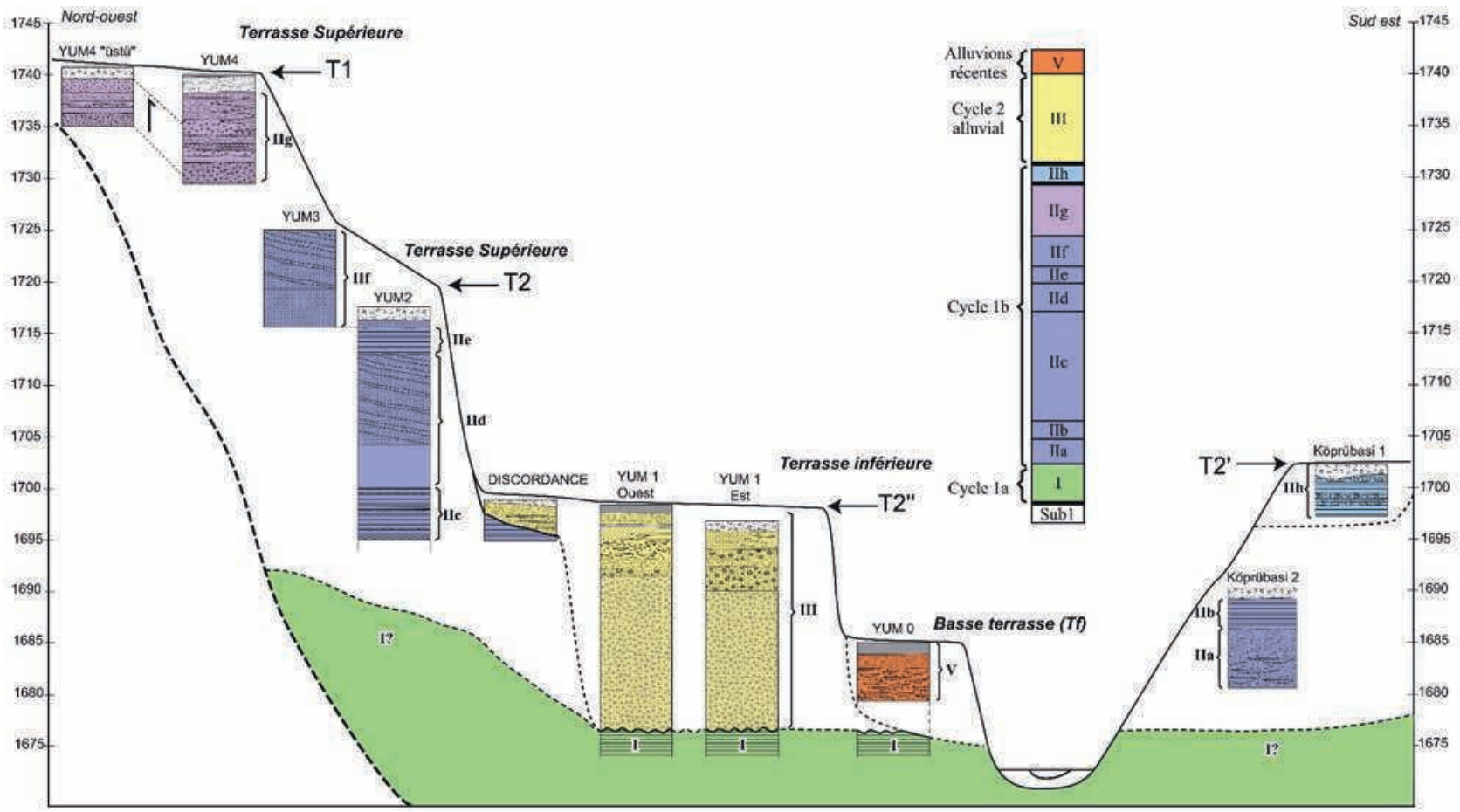

Fig. 6: Stratigraphie de l'amont de la Karasu.

I. Argiles et limons laminés lacustres; II a. Sables avec lentilles grossières à la base, unité de transgression; II b. Limons fins laminés; II c .Alternance limons laminés avec passages plus grossiers et ripple marks; II d. Foresets avec alternances de lits plus ou moins grossiers (sables et graviers) ; II e. Limons fins laminés avec lits épais; II f. Foresets avec alternances de lits plus ou moins grossiers (sables et graviers) ; II g. Unité à faciès littoraux :alternance galets, sables et limons et passages latéraux; II h. Limons et sables fins laminés alternant avec lits à sismites; III. Séquence fluviatile avec alternance de sables à stratification entrecroisée et limons; V. Unité fluviatile grossière recouverte par limons de débordement; I. Unités lacustres à argiles et limons laminés.

Fig. 6: Stratigraphy of the upstream course of the Karasu River:

I. Lacustrine clays and laminated silts; II a. Transgressive unit with sands and coarser features at its base;

II b. Thinly laminated silts; II c. Laminated silts alternating with coarser beds, exhibiting ripple marks; II d. Foresets with thin and coarser beds (sands and gravels); II e. Thin laminated silt with thick beds; II f. Foresets with thin and coarser beds (sands and gravels); II g. Unit with shore faciès, pebbles and sands, changing laterally; II h. Laminated silts and thin sands alternating with sismite-deformed beds; III. Fluviatile sequence with cross bedded sands alternating with silty and thick beds; $V$. Coarse fluviatile unit covered by flood silts.

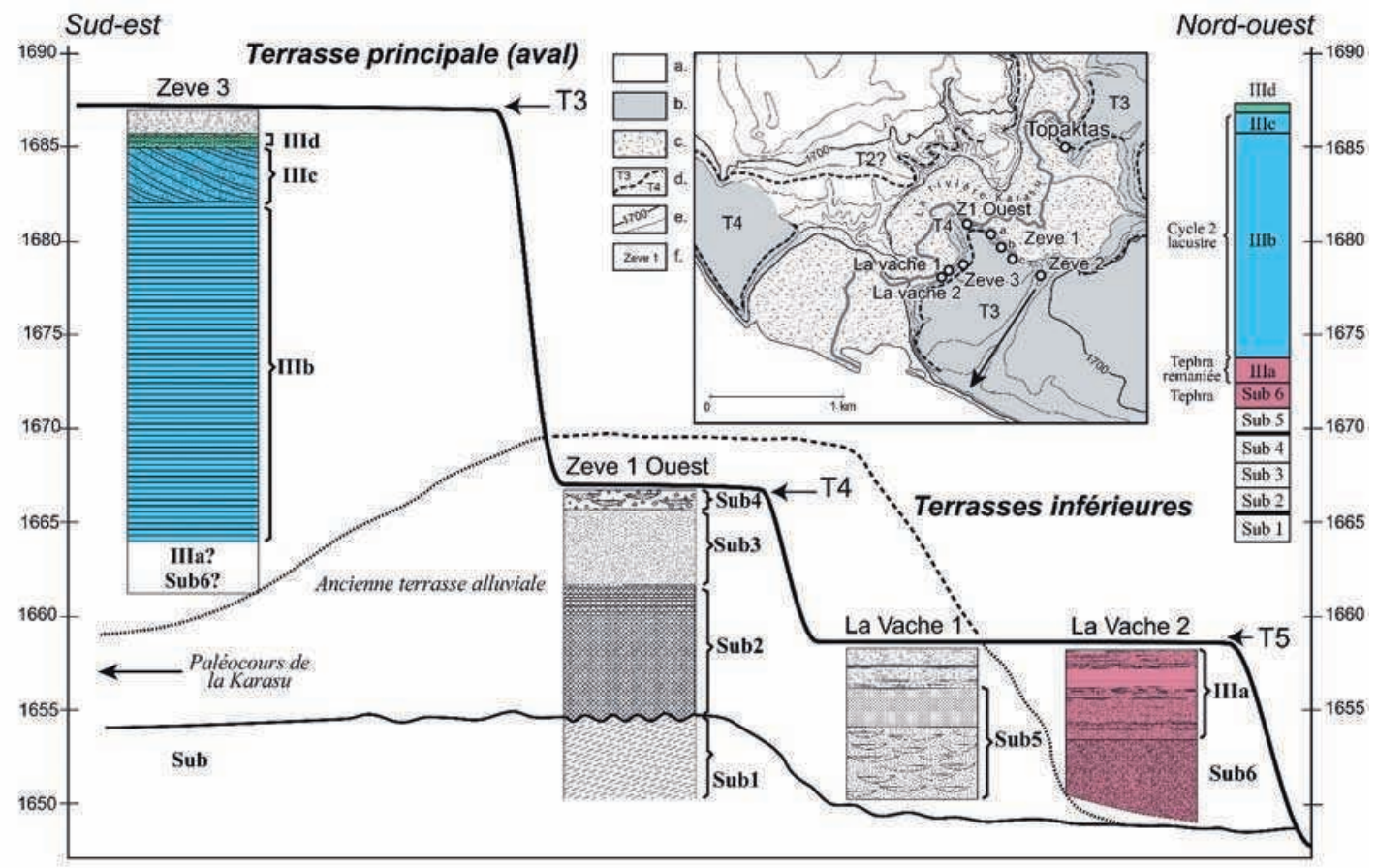

Fig. 7 : Carte géomorphologique et stratigraphie de l'aval de la Karasu.

a. Substrat; b. Dépôts du Cycle 2; c. Remplissage alluvial post-Cycle 2; d. Limites entre les surfaces de terrasses; e. Fond topographique $1 / 25$ 000 ; f. Noms des coupes étudiées.

Fig. 7: Geomorphological map and stratigraphy of the downstream course of the Karasu River.

a. Substratum; b. Lacustrine Cycle 2 deposits; c. Post lacustrine Cycle 2 alluvial fill; $d$. Terrace surface limits; e. Contour lines; f. Names of sections studied. 


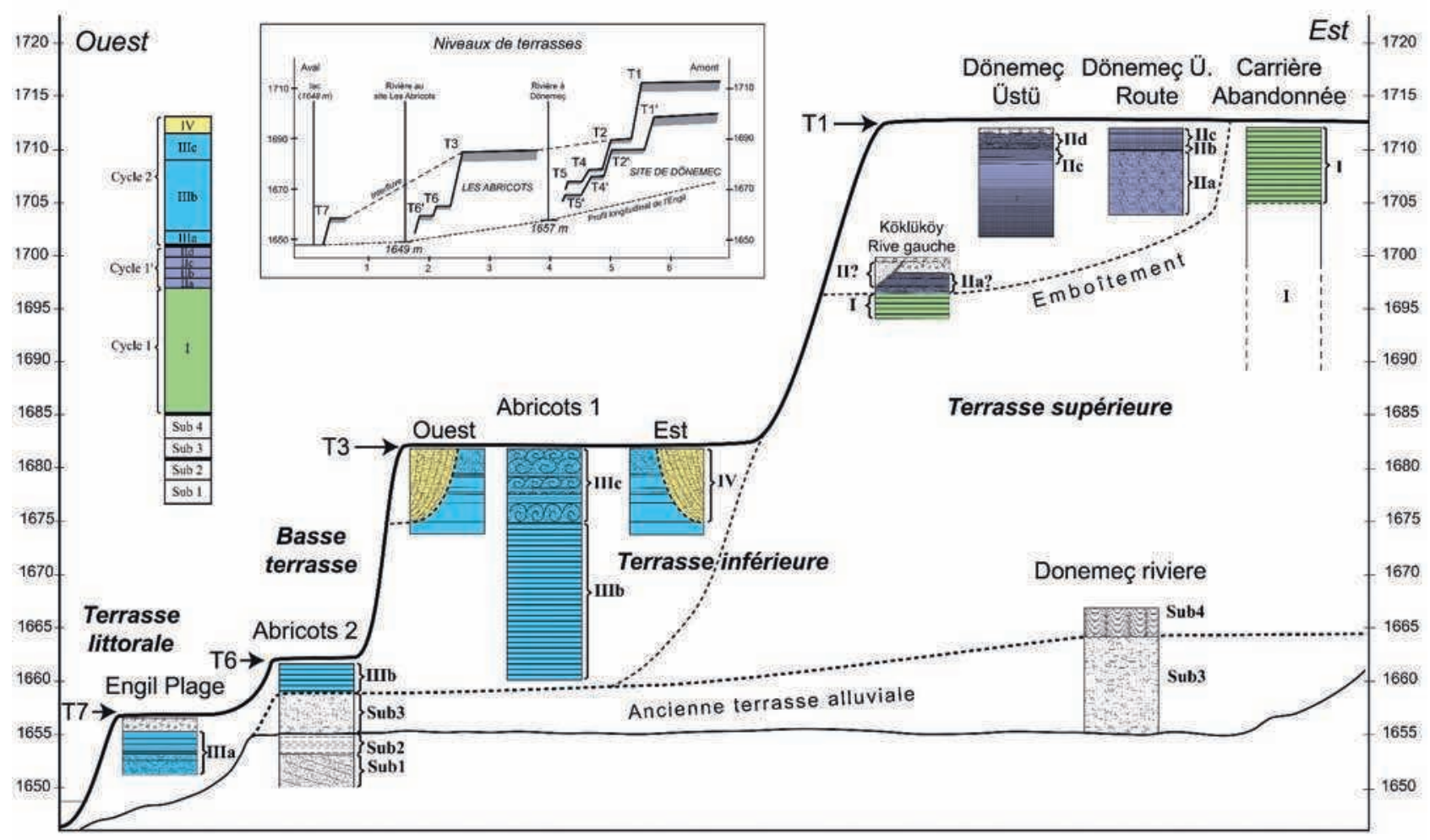

Fig. 8: Niveaux de terrasses et stratigraphie de la vallée de l'Engil.

Sub1. Sables; Sub2. Argiles; Sub3. Conglomérats; Sub4. Stromatolites; I. Limons laminés et argiles lacustres;

II a. Sables fins (avec sismites?); II b. Lits sableux fins à stratification entrecroisée; II c. Sables laminés;

II d. Sables avec stratification ondulée; III a. Sables, sables fins et limons; III b. Argiles et limons laminés ;

III c. Unités sableuses et silteuses avec lit à sismites épais; IV. Remplissage alluvial avec foresets sableux.

Fig. 8: Terrace levels and stratigraphy of the Engil Valley.

Sub1. Sands; Sub2. Clays; Sub3. Conglomeratic sands and pebbles; Sub4. Stromatolites; I. Lacustrine laminated silts and clays; II a. Thin sands (with sismites?); II b. Cross-bedded sands; II c. Laminated sands; II d. Sands with wavy stratification; III a. Units with sands and thin sands and silts; III b. Laminated clays and silts; III c. Sandy and silty units with thick sismite beds; IV. Alluvial fill with sandy foresets.

coupe Köprübasi) soulèvent une interrogation: correspondent-ils à un niveau de régression intermédiaire (alluvial) entre deux transgressions ou à un sédiment d'avant-plage? Au site de Köklüköy la partie occidentale de la séquence confirme l'emboîtement indiqué par une incision comblée par des dépôts alluviaux et des dépôts deltaïques fins inclinés (fig. 8).

En aval, au site «Les Abricots», les dépôts inférieurs sont des limons lacustres laminés accumulés sur $15 \mathrm{~m}$, qui reposent sur le substrat géologique (conglomérats sur argiles). Cette série transgressive est surmontée par $10 \mathrm{~m}$ d'une série sableuse affectée par d'importantes sismites (fig. 8). La transition d'un lac profond à un lac peu profond se fait progressivement, comme le montre l'alternance de niveaux limono-sableux laminés avec des niveaux sableux dans la partie médiane de la coupe. Aux Abricots 1, une incision similaire à celle du site de Köklüköy et profonde d'environ $6 \mathrm{~m}$ est enregistrée sous le sommet de la séquence. Les dépôts sont les mêmes qu'à Köklüköy: des limons disposés en foresets raccordés à un ancien niveau d'eau.

Plus en aval encore, la falaise qui surplombe le littoral du lac actuel $(+13 \mathrm{~m})$ tranche des dépôts lacustres sablolimoneux correspondant au début d'une série transgressive.

Les positions altimétriques ainsi que la variété des types de contacts entre les unités limoneuses et sableuses montrent une évolution complexe caractérisée par la succession de nombreuses variations du niveau d'eau d'amplitude relativement faible.

\subsection{2 - Identification des terrasses}

Dans la vallée de l'Engil, tant à l'amont (Dönemeç) qu'à l'aval (Les Abricots), les terrasses correspondent à des surfaces d'érosion qui recoupent à la fois les remplissages lacustres et des formations de travertins (rive gauche). A l'amont, le nombre de niveaux de terrasses s'accroît, leurs surfaces recoupant une seule série lacustre transgressive.

Le niveau supérieur (T1) est une vaste terrasse à $1712 \mathrm{~m}$ (1699 m en rive gauche) qui tronque des dépôts de nature différente. Il s'appuie sur un épais travertin en rive droite et se termine par un talus décamétrique moins marqué en rive gauche. Le second niveau (T2), intermédiaire, à $1690 \mathrm{~m}$ (1686 m en rive gauche), est de plus faible extension spatiale. Son talus, de quelques mètres sur les deux rives de la rivière, tronque les dépôts lacustres formant la base de l'accumulation conservée dans la terrasse supérieure. Ces terrasses de la partie amont de la vallée de l'Engil dans le secteur de Dönemeç sont appariées; leur formation à différentes altitudes de la vallée est liée à celle des méandres (érosion plus ou moins forte des rives) et à la divagation de la rivière lors de l'incision de la vallée (également contrainte par le fonctionnement 
tectonique de la vallée). Dans le fond de la vallée, une quatrième terrasse, plus basse (T5: $1667 \mathrm{~m}$ ), correspond au toit d'une formation stromatolitique recouvrant des conglomérats anciens émergeant sous le remplissage lacustre transgressif.

La transition avec les sites plus en aval se caractérise par la disparition des niveaux topographiques intermédiaires et par la présence d'un niveau de terrasse principal incliné, T3. En aval l'altitude de ce niveau est de $1685 \mathrm{~m}$ (fig. 8), et se raccorde vers l'amont à T2/T2' (1686-1690 $\mathrm{m})$. Le rebord de cette terrasse forme un talus de $25 \mathrm{~m}$ de hauteur, tant en rive gauche qu'en rive droite. Jusqu'au lac, le lit majeur de la rivière est dominé par le prolongement du niveau T3. L'aval de l'Engil est l'un des rares secteurs où la falaise littorale est reliée topographiquement au système de terrasses de la vallée (T3).

\subsection{3 - Interprétations morpho-stratigraphiques}

Les unités lacustres conservées sous les terrasses de Dönemeç (I) témoignent d'une transgression lacustre ancienne (Cycle 1) qui a immergé toute la vallée jusqu'à atteindre une altitude supérieure à $1712 \mathrm{~m}$. La stratigraphie montre que ces unités ont été tronquées lors d'une première régression assez rapide jusqu'à une altitude d'environ 1697 m (contact supérieur I/IIa à Köklüköy). Une remontée postérieure du niveau du lac à une altitude supérieure à 1712 m explique le dépôt d'unités plus sableuses (sables grossiers à la base) et pour certaines déformées, IIa, IIb, IIc et IId.

Sur la base de ces différences de faciès et d'altitude, on peut distinguer deux séquences, celles de Dönemeç (unités I-II : Cycle 1) et des Abricots (unités III : Cycle 2), qui correspondent à deux transgressions différentes, similaires aux deux grands cycles lacustres identifiés dans la vallée de la Karasu. En effet, la série lacustre IIIc et les unités supérieures plus sableuses (IIId) sont emboîtées dans la série I-II, et sont donc postérieures. Les unités sablo-limoneuses de la terrasse littorale forment, en fait, la base de la séquence transgressive III (Cycle 2).

Au site «Les Abricots 1 », le remplissage limoneux (IV) du chenal incisé dans l'unité IIId témoigne d'une oscillation du niveau d'eau (abaissement du lac-incision du réseau hydrographique; puis remontée du niveau du lac et colmatage des chenaux). La régression lente qui a suivi a provoqué l'abrasion progressive de la surface de la terrasse T3. Une phase d'abaissement rapide de la nappe d'eau a ensuite conduit à l'entaille des formations du substrat avec un palier à $1660 \mathrm{~m}$ («Les Abricots $2 »)$ puis une reprise de l'incision jusqu'à une altitude que nous n'avons pas pu déterminer parce qu'inférieure à celle du rivage actuel.

\section{5 - CORRÉLATIONS}

\section{1 - CYCLES LACUSTRES ET CHRONOLOGIE}

Dans les parties anciennement ennoyées des vallées du pourtour du lac (Zilan, Kirklar-Muradiye et Kotum), la stratigraphie des dépôts lacustres suggère l'emboîtement de deux séries sédimentaires correspondant à deux grands cycles lacustres.

Cycle 1b: Les coquilles de Dreissena provenant des dépôts littoraux du maximum atteint par la transgression du Cycle 1 à Yumrutepe (YUM4: $1740 \mathrm{~m}$ ) ont livré des âges ${ }^{14} \mathrm{C}>31$ et 34 ka BP (Kuzucuoğlu et al., 2008b). Dans la vallée de Kotum ( $c f$. fig. 1), au sud-ouest du lac, un travertin contemporain ou immédiatement antérieur à la phase initiale de la transgression du Cycle $1 \mathrm{~b}$ a été daté $102.2 \mathrm{ka}$ (Kuzucuoğlu et al., 2008b). Cette date est à prendre avec précautions étant donné la nature de la formation datée et les intervalles de temps importants notamment pour la base du travertin (tab. 2). D'autre part, une retombée ponceuse fossilisée par cette même séquence transgressive a été datée par ${ }^{39} \mathrm{Ar} /{ }^{40} \mathrm{Ar}$ de 117.8 $\pm 5.2 \mathrm{ka}$. L'âge ${ }^{39} \mathrm{Ar} /{ }^{40} \mathrm{Ar}$ de la retombée correspond aux périodes d'activité du Nemrut datées par Çubukçu (2008). Dans cette vallée de Kotum, le sommet (1725 m) de la haute terrasse formée par ces dépôts du Cycle 1 est équivalent de celui de la terrasse T2 de la Karasu et de l'Engil (Mouralis et al., 2010).

Ces deux dates indiquent que la transgression lacustre du Cycle $1 \mathrm{~b}$ est postérieure à l'Interglaciaire Eemien. Cet âge confirme les estimations précédentes faites par Degens et al., (1978), Landmann et al. (1996) et Kempe et al. (1997) qui ont considéré que les dépôts de terrasses autour du lac de Van avaient un âge couvrant le dernier Glaciaire et que l'âge du lac lui-même se situerait entre 100 et 60 ka BP (Kempe et al., 2002).

Cycle 2: A Topaktas (secteur légèrement en amont du site de Zeve), une retombée ponceuse de $4 \mathrm{~m}$ d'épaisseur a été datée $\operatorname{par}{ }^{39} \mathrm{Ar} /{ }^{40} \mathrm{Ar}$ de $31.4 \pm 6.6 \mathrm{ka}$ (Kuzucuoğlu et al., 2010; Mouralis et al., 2010). Cette retombée, recouverte par la séquence transgressive du cycle 2 (unités III) dans la coupe de la Vache 1 (fig. 7), pré-date donc la deuxième grande transgression (Cycle 2). L'âge du niveau maximum atteint par cette transgression a été déterminé au nord-est du lac, au site de Kirklar (localisation fig. 1), où un niveau organique à $1700 \mathrm{~m}$ situé en haut de la séquence transgressive continue a été daté par ${ }^{14} \mathrm{C}$ de $20780 \pm 650 \mathrm{ka} \mathrm{BP}$ et $21375 \pm 425 \mathrm{ka} \mathrm{BP}$ (tab. 2). Calibrées, ces dates deviennent 24 et 26 cal ka BP. La transgression du Cycle 2 a donc débuté après $30 \mathrm{ka} \mathrm{BP}$, recouvrant le tephra important de Topaktas. Elle a atteint son maximum vraisemblablement vers 26 ka BP c'est-àdire peu avant le DMG. Dans la vallée de l'Engil au site Les Abricots 1, Kempe et al. (2002) ont daté une deuxième pulsion transgressive (III, Cycle 2) avec une mise en place des niveaux transgressifs inférieurs à partir de 23 cal BP $( \pm 19.5$ ka non cal BP), le maximum de la terrasse à cet endroit ( $>1688 \mathrm{~m}:+40 \mathrm{~m}$ ) étant atteint vers $22.5 \mathrm{cal} \mathrm{BP}$ ( $\pm 19 \mathrm{ka}$ non cal BP). Or, peu avant 23 cal BP, le niveau du lac était à $1666 \mathrm{~m}$ (Kempe et al., 2002). L'ensemble de ces résultats permettent de reconstituer une transgression en deux phases avant puis pendant le DMG; ils illustrent également la rapidité de chacune de ces hausses du niveau du lac (20-25 m au moins en mille ans). 


\begin{tabular}{|c|c|c|c|c|c|c|}
\hline \multicolumn{7}{|c|}{${ }^{14} \mathrm{C}$} \\
\hline Année & $N^{\circ}$ Gif/Artemis & Description & Age & Incertitude & Delta ${ }^{13} \mathrm{C}$ & $\begin{array}{l}\text { Ages } \\
\text { calibrés }\end{array}$ \\
\hline 2008 & GIF 12320 & $\begin{array}{l}\text { Kirklar niveau supérieur } \\
\text { (niveau humifère) }\end{array}$ & 20780 & 650 & & $24 \mathrm{cal} \mathrm{ka} \mathrm{BP}$ \\
\hline 2008 & GIF12138 & $\begin{array}{l}\text { Kirklar niveau inférieur } \\
\text { (niveau humifère) }\end{array}$ & 21375 & 425 & $-26,78$ & $26 \mathrm{cal} \mathrm{ka} \mathrm{BP}$ \\
\hline \multicolumn{7}{|c|}{ U/Th } \\
\hline Année & $\mathrm{N}^{\circ}$ Echantillon & \multicolumn{2}{|l|}{ Description } & \multicolumn{3}{|c|}{ Age } \\
\hline 2007 & VAN 06 33a & \multicolumn{2}{|c|}{$\begin{array}{c}\text { Travertin-niveau supérieur } \\
\text { Kotum (aval) }\end{array}$} & \multicolumn{3}{|c|}{$102.2(-3.7 ;+3,8) \mathrm{ka}$} \\
\hline 2007 & VAN 06 33b & \multicolumn{2}{|l|}{$\begin{array}{l}\text { Travertin-base } \\
\text { Kotum (aval) }\end{array}$} & \multicolumn{3}{|c|}{$102.1(-7.5 ;+8.1) \mathrm{ka}$} \\
\hline \multicolumn{7}{|c|}{ ArlAr } \\
\hline Année & $\mathrm{N}^{\circ}$ Echantillon & \multicolumn{2}{|l|}{ Description } & \multicolumn{3}{|c|}{ Age } \\
\hline 2008 & VAN 021 & \multicolumn{2}{|c|}{$\begin{array}{l}\text { Retombée plinienne } \\
\text { Kotum (aval) }\end{array}$} & \multicolumn{3}{|c|}{$117.0+/-5.2 \mathrm{ka}(2 \sigma)$} \\
\hline 2008 & VAN 030 & \multicolumn{2}{|c|}{$\begin{array}{l}\text { Retombée plinienne } \\
\text { Topaktas (Karasu aval) }\end{array}$} & \multicolumn{3}{|c|}{$31.4+/-6.6 \mathrm{ka}(2 \sigma)$} \\
\hline
\end{tabular}

Tab. 2 : Tableau des résultats pour les datations.

Tab. 2: Table of the datation results.

\section{2 - CYCLES LACUSTRES, TERRASSES ET AJUS- TEMENT DU PROFIL DES RIVIÈRES}

Un certain nombre de points communs entre les deux vallées, identifient les grands traits d'un système de terrasses développé pourtant dans des contextes géologiques et structuraux différents. Ce système est organisé avec de hautes terrasses, à l'amont incisées en plusieurs replats, tandis que dans les parties aval des vallées, hormis quelques très basses et petites terrasses toujours localisées près des embouchures, une large terrasse moyenne domine la topographie. Les terrasses sont sous-tendues par des dépôts de faciès différents emboîtés, tronqués par une surface d'érosion: ce sont donc des terrasses de régression. Stratigraphie et faciès ont permis d'établir des corrélations entre les secteurs amont et aval des vallées.

Deux grands cycles transgressifs sont chacun associés à plusieurs terrasses.

Le plus ancien des cycles, le Cycle 1, correspond en fait à une double transgression (Cycles $1 \mathrm{a}$ et $1 \mathrm{~b}$ : fig. 9), qui ont chacune atteint des altitudes très élevées. Si le niveau maximum du Cycle 1a n'a pas pu être identifié, celui du Cycle $1 \mathrm{~b}$ a été déterminé à Yumrutepe à $1740 \mathrm{~m}$, soit +90 m au-dessus du niveau actuel. Etant donné l'existence de failles affectant ces dépôts (YUM 4 Üstü) (fig. 6), il est possible que cette amplitude ait une composante tectonique locale. L'impact de la tectonique est confirmé par l'élévation anormale des dépôts littoraux à Toki (localisation: fig. 1) où ils ont été mesurés à $1751 \mathrm{~m}(+101 \mathrm{~m}$ au-dessus du lac actuel, soit $16 \mathrm{~m}$ au-dessus du seuil topographique actuel du bassin du lac). Les séquences transgressives de ces deux pulsations anciennes se caractérisent par des dépôts varvés argileux pour le cycle 1a (par exemple à «Dönemeç Carrière-abandonnée »: 1710$1712 \mathrm{~m}$ ), et par les couches limoneuses et deltaïques pour le cycle 1b (par exemple à YUM2: $1725 \mathrm{~m}$ ).
La hausse du niveau d'eau lors de la transgression du Cycle 1b s'est réalisée par paliers, signalant des souscycles enregistrés par les deux cônes deltaïques séparés par des limons lacustres à Yumrutepe (YUM 3). Confortant la complexité des fluctuations des niveaux du lac durant le Cycle 1, le site de Köprübasi et la grande coupe de Dönemeç Üstü (II) témoignent du sursaut transgressif après le début de la régression intermédiaire (fig. 9). Le niveau atteint par le lac lors de cette transgression intermédiaire a été de quelques mètres supérieurs à l'altitude des dépôts sableux soit 1702-1712 m (fig. 9).

Pendant la transition entre les deux grands cycles, une forte incision a précédé l'emboîtement de dépôts alluviaux très épais (YUM 1) dont les couches terminales (limons de crue) reposent en discordance sur les bottomsets érodés du Cycle 1b (YUM2). A Yumrutepe, l'incision initiale a atteint dans les bottomsets érodés l'altitude de $1677 \mathrm{~m}$ soit 5 mètres au-dessus du cours actuel de la rivière. Dans l'hypothèse où le profil en long de la paléoKarasu était similaire à celui d'aujourd'hui, le niveau du lac correspondant à cette incision devait donc se trouver à une altitude similaire à l'actuelle, soit vers $1650 \mathrm{~m}$.

La deuxième transgression (Cycle 2) pénètre dans des vallées où elle submerge des lits majeurs plus larges qu'aujourd'hui. Tandis que se déposent à l'aval, dans un milieu de lac relativement profond, les limons de Zeve 3 et que le niveau de base remonte, la paléoKarasu continue à délivrer à l'amont une quantité importante d'alluvions grossières (III, fig. 6). En effet, cette deuxième transgression ne modifie que peu aggradation alluviale continue à Yumrutepe où elle n'est pas recouverte de dépôts lacustres. La rivière, alors, possède une énergie assez importante et son profil longitudinal s'aplatit en s'ajustant au nouveau niveau de base qui se stabilise vers 1689-1692 m (Zeve 1: unité IIIc; «Les Abricots 1»: unité IIIe), soit $+42 / 45 \mathrm{~m}$ au-dessus du 


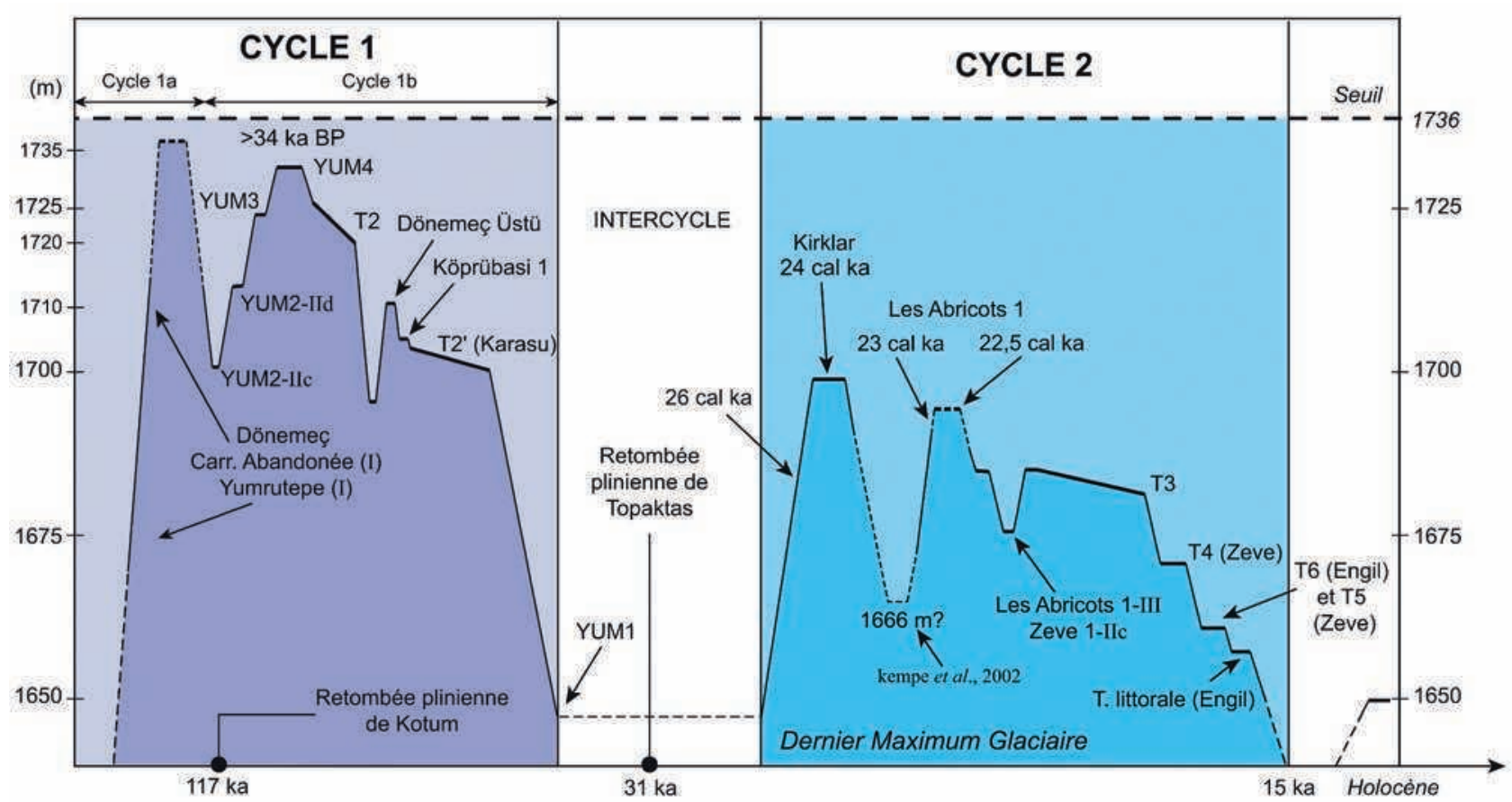

Fig. 9 : Courbe de la variation du niveau du lac durant le Pléistocène supérieur. Fig. 9: Curve of the Lake Van level variations during the Upper Pleistocene.

niveau actuel. Ce modèle morphosédimentaire suggère donc une pénétration du lac d'environ $2 \mathrm{~km}$ vers l'amont par rapport au rivage actuel.

Le lac s'est ensuite retiré à un niveau d'environ $1675 \mathrm{~m}$ (base des chenaux au sommet des Abricots 1 et de T5 à Zeve), avant de remonter à une altitude supérieure à $1688 \mathrm{~m}$ (sommet du remplissage III). La seconde oscillation du cycle 2 conduit donc à un niveau maximum similaire à celle enregistrée lors de la première oscillation.

Lors de la régression qui suit les maxima du Cycle 2, le lac s'est retiré à un niveau beaucoup plus bas que l'actuel, comme en témoigne l'encaissement des rivières qui a abouti à la formation de basses terrasses d'érosion recoupant les dépôts des Cycles 1 et 2 ainsi qu'à l'incision du substrat à Zeve (Zeve 1, Ravin La Vache 1 et 2, Çitören), et dans l'Engil (Les Abricots 2).

Aujourd'hui, la morphologie des lits majeurs dans les parties aval des vallées correspond à un remplissage alluvial postérieur important, en réponse à une dernière remontée du lac (début d'un troisième cycle) jusqu'au niveau actuel (1645-1650 m).

\section{3 - IMPACT DE LA TECTONIQUE}

Quelques différences entre les deux vallées viennent nuancer ce schéma général. Il s'agit principalement de niveaux repères corrélés par le faciès et la stratigraphie, mais dont les variations d'altitudes indiquent l'influence locale probable de la tectonique (soulèvements). La mesure DGPS a permis de fixer l'altitude du seuil actuel du lac à 1735 m (Christol et al., 2008b; Kuzucuoğlu et al., 2008), altitude au-dessus de laquelle les eaux du lac se déverseraient aujourd'hui dans le bassin du Tigre au sud-ouest du lac. A YUM 4, les dépôts littoraux de la transgression du Cycle $1 \mathrm{~b}$ atteignent $1740 \mathrm{~m}$ d'altitude. A Toki sur l'autre rive, ils forment une falaise rectiligne où ils présentent une altitude de $1751 \mathrm{~m}$. Seul un soulèvement peut expliquer ces altitudes trop hautes par rapport au seuil actuel du lac. Le rejet vertical serait de 5 à $16 \mathrm{~m}$ environ. Néanmoins, des varves argileuses positionnées encore plus haut dans la vallée de la Zilan (Kuzucuoğlu et al., sous presse) et correspondant vraisemblablement au maximum atteint lors du Cycle 1a, témoignent d'un seuil très ancien plus haut que l'actuel. Ce seuil est à mettre en relation avec les coulées pyroclastites qui ont, avant la retombée plinienne datée de $117 \mathrm{ka}$, remplit les vallées du secteur sud-oriental, barrant le lac. L'érosion des faciès meubles de ces pyroclastites a abaissé le seuil (Mouralis et al., 2010). Dans les vallées de la Karasu et de l'Engil, le décalage altimétrique entre les dépôts du Cycle $1 \mathrm{~b}$ (une dizaine de mètres) semble, lui, s'expliquer plutôt par la tectonique, avec le jeu des failles affectant les dépôts. Les signatures altimétriques de la tectonique ont affecté la terrasse supérieure, et non pas la terrasse inférieure. Ces mouvements sont donc datables de la transition entre Cycles 1 et 2 . Cette activité tectonique est restée locale, déterminée par une faille située au nord de Van, qui a affecté les formations amont de la vallée de la Karasu (Kuzucuoğlu et al., sous presse).

\section{4 - HAUTS NIVEAUX ET ANCIENS VOLUMES LACUSTRES}

Avant la première transgression repérée dans le bassin de Van (Cycle 1a), le niveau du lac se positionne sans doute nettement en dessous du niveau actuel puisque le lac devait alors posséder un exutoire vers l'ouest. 
$\mathrm{La}$ transgression du Cycle $1 \mathrm{~b}$ qui a atteint $1730 \mathrm{~m}$ d'altitude a concerné, elle un volume d'eau de $960 \mathrm{~km}^{3}$ (à comparer avec le volume actuel de $615 \mathrm{~km}^{3}$ ) pour une surface de $4730 \mathrm{~km}^{2}$ (rejet de faille éliminé). Cette hausse de niveau équivaut donc à $350 \mathrm{~km}^{3}$, soit environ 0,5 fois le volume actuel. Pendant la régression qui a suivi le Cycle 1, le volume atteint a probablement été équivalent à l'actuel; la régression n'en signifie pas moins que le lac a perdu environ 0,5 fois le volume actuel d'eau. Les indices morphosédimentaires suggèrent que cette régression s'est faite relativement lentement au début. Un premier niveau de régression à $1690 \mathrm{~m}$ environ $(+42 \mathrm{~m}) \mathrm{a}$ été causé par une baisse du volume de $180 \mathrm{~km}^{3}$. La transgression intermédiaire (IIh) suivante, durant laquelle le lac a atteint 1705-1715 m (+53 m/ + $63 \mathrm{~m})$ correspond à un volume de $842-892 \mathrm{~km}^{3}$.

$\mathrm{Au}$ maximum de la seconde transgression (Cycle 2) le lac a atteint un niveau d'au moins 1690/1700 m soit + 40/50 m au-dessus du niveau actuel; cette altitude correspond à un volume de $773 \mathrm{~km}^{3}$. Entre la fin de la régression inter-Cycles 1 et 2 , et le maximum atteint par la transgression du Cycle 2, le volume du lac a augmenté de $160 \mathrm{~km}^{3}$.

\section{6 - DISCUSSION}

L'apport d'eau considérable impliqué par la première grande transgression identifiée peut s'expliquer, d'un point de vue climatique, soit par une forte baisse de l'évaporation, soit par l'augmentation des précipitations dans le bassin versant, soit par les deux facteurs combinés. L'hypothèse d'une origine exclusivement climatique des variations de niveau du lac est discutable. Un climat plus humide et/ou plus nuageux (donc moins propice à de forts taux d'évaporation) est envisageable. Dans ce cas, l'homogénéité et la continuité des séquences sédimentaires de la transgression du Cycle $1 \mathrm{~b}$ suggère une réponse à un changement climatique brutal. Or, le climat du début du dernier glaciaire ne semble pas correspondre à ces caractéristiques d'augmentation rapide de l'humidité. Par conséquent il apparaît peu probable que le climat soit seul à l'origine de la hausse du niveau du Cycle $1 \mathrm{~b}$ vers 115-110 ka.

Par ailleurs, des changements paléogéographiques dans le bassin versant semblent avoir accompagné la transgression et les régressions du Cycle 1 ( $a$ et $b$ ). En effet, des modifications de réseau hydrographique ont été identifiées, dont les causes sont soit l'activité tectonique (isolement du bassin du lac Erçek), soit l'activité volcanique (vallées du sud-ouest du lac), soit l'érosion régressive en contexte karstique (vallées nord du massif de Bitlis) (Kuzucuoğlu et al., 2010; Mouralis et al., 2010). Ainsi, la transgression du Cycle 1a pourrait avoir été causée par la fermeture du lac consécutive à de très abondantes coulées pyroclastiques ponceuses (partiellement indurées) émises par le Nemrut; celle du Cycle 1b pourrait, elle, avoir été causée par un deuxième barrage formé par une coulée ignimbritique ultérieure associée à la retombée ponceuse datée $117 \mathrm{ka}$ (Mouralis et al.,
2010). En effet, les indices morphosédimentaires indiquent que la transgression du Cycle 1a, elle aussi, a été relativement rapide, et que la période de haut niveau a été également assez courte (fig. 9).

Les oscillations du Cycle $1 \mathrm{~b}$ semblent, elles, répondre à des variations climatiques dans un contexte d'absence d'exutoire. Par exemple, alors que l'équilibre exprimé par le haut niveau de YUM4 est de courte durée, la régression qui s'ensuit et qui atteindra au moins 55 à $60 \mathrm{~m}$, pourrait répondre à l'assèchement du climat pendant le dernier Glaciaire. Parallèlement cependant, cette aridification pourrait aussi avoir favorisé la soustraction de certaines têtes de rivières et leurs affluents aujourd'hui capturés par le bassin du Tigre dans les secteurs karstiques du massif de Bitlis (Kuzucuoğlu et al, 2010). Quoi qu'il en soit cette grande régression inter-cycles est vraisemblablement due à l'ajustement du système lacustre au climat du dernier glaciaire caractérisé par une forte évaporation et de faibles précipitations. Cette période reste aujourd'hui mal délimitée chronologiquement bien que l'âge de $30 \mathrm{ka}$ BP obtenu à Topaktas en positionne la fin.

En effet, la transgression du Cycle 2 débute entre $30 \mathrm{ka}$ cal BP (Topaktas) et $26 \mathrm{ka}$ cal BP; elle atteint son premier maximum vers $26 \mathrm{ka} \mathrm{cal} \mathrm{BP} \mathrm{(Kirklar).} \mathrm{Après} \mathrm{une}$ régression de $25 \mathrm{~m}$ environ entre 24 et $23 \mathrm{ka}$ cal BP, le lac remonte à nouveau de $25 \mathrm{~m}$ environ entre 23 et $22 \mathrm{ka} \mathrm{cal}$ BP (Kempe et al., 2002). Ces changements de niveau qui composent le Cycle 2 semblent répondre à des changements climatiques, discutés dans Landmann et al. (1996). En Anatolie, la période 28-20 ka cal BP correspond à une phase de hauts niveaux lacustres datée dans de très nombreux lacs anatoliens (Kuzucuoğlu \& Roberts, 1998; Fontugne et al., 1999). Cette phase existe également au Proche Orient, avec un haut niveau dans la Mer Morte entre $25 \mathrm{ka}$ et $20 \mathrm{ka}$ BP (Bartov et al., 2002), et dans le lac Kinneret autour de 25 ka BP (Hazan, 2005). Le bilan hydrologique excédentaire de ces lacs est expliqué, par les auteurs, par une diminution importante de l'évaporation elle-même liée à différents facteurs: augmentation de la couverture nuageuse, baisse des températures (l'été notamment). La baisse du niveau d'eau qui intervient dans le lac de Van vers $21 \mathrm{ka}$ cal BP (= fin du maximum du Cycle 2), et qui s'interrompra à un niveau nettement inférieur à l'actuel, est, elle aussi, parallèle avec les baisses de niveau et assèchements de lacs que connaissent les lacs anatoliens et du Proche-Orient à la fin du DMG. Ces régressions sont généralement interprétées comme la réponse des lacs à une évaporation croissante (Kuzucuoğlu \& Roberts, 1998).

\section{7 - CONCLUSION}

L'étude morphosédimentaire des terrasses du pourtour du lac de Van permet de positionner les altitudes atteintes par d'anciennes lignes de rivage et de caractériser les modalités des changements de niveau d'eau du lac. Elle autorise l'établissement d'une courbe des variations de niveau lacustre pendant le Pléistocène supérieur. 
Les amplitudes de ces variations supposent des fluctuations de volumes très importantes par rapport au volume actuel et posent la question de l'origine des changements du bilan hydrologique.

A l'intérieur de deux grands cycles lacustres, la géométrie des enregistrements morphosédimentaires témoigne de variations fines des milieux de sédimentation, donc d'oscillations de la cote du lac. Les deux cycles identifiés ne sont pas tout à fait comparables. Le Cycle 1 est «dédoublé » avec d'une part la grande transgression de Yumrutepe-Dönemeç Carrière abandonnée (I) et d'autre part la transgression de Yumrutepe-Köprübasi (II). Cependant, les transgressions se sont produites rapidement, et les très hauts niveaux atteints ne sont pas restés stables longtemps (Cycle 2, les Abricots 1). Le déroulement des transgressions qui ont commencé à partir de niveaux inférieurs à l'actuel, apparaît moins complexe que celui des régressions qui, plus lentes, se sont produites par étapes. Chacun des deux grands cycles identifiés a connu une oscillation initiale immédiatement après avoir atteint son maximum (fig. 9). La longue durée et la descente par étape pendant les régressions ont façonné les surfaces planes des terrasses (plans de régression lacustre).

Si la hausse de $+85 \mathrm{~m}$ qui affecte le niveau du lac au début du Cycle 1b intervient dans un contexte paléogéographique complexe (qui voit très probablement la fermeture de l'exutoire par un barrage volcanique), celle de +50 m observée au début du Cycle 2 et qui est datée du DGM, répond à un changement climatique également responsable de mouvements similaires dans les autres lacs anatoliens et du Moyen Orient. En outre, pendant le Pléistocène supérieur, l'activité tectonique et volcano-tectonique a été importante, modifiant les dépôts des terrasses, où nous avons observé des déformations verticales et des sismites. Rejeux de failles et soulèvements ont été principalement localisés soit le long de la faille S-N qui borde la plaine occupée par la ville de Van; soit en relation avec les failles bordières des grabens occupés par les principales vallées affluentes du lac (Engil, Karasu et Zilan).

Les jalons chronologiques présentés ici sont les premiers de l'histoire pléistocène du lac de Van; ils demeurent, bien sûr, incomplets. Au-delà de la reconstitution des anciens niveaux lacustres et de la recherche de leurs causes, les questions de l'origine du lac de Van et de son âge, des changements paléohydrologiques dans le bassin versant (addition/soustraction de bassins versants secondaires), des variations holocènes du niveau $\mathrm{du}$ lac... restent des questions dont les enjeux scientifiques sont fondamentaux pour la connaissance de l'évolution climatique et environnementale de l'Anatolie et du Proche-Orient.

\section{REMERCIEMENTS}

Les auteurs remercient les institutions qui ont soutenu le projet «ANOVAN» (2006-2008): le TÜBITAK (ÇAYDAG 105Y125; SOBAG 105Y127), le CNRS (ECLIPSE II et PICS), l'Université de Van (FED-B-10), le Ministère des Affaires Etrangères français (PHC) et l'ambassade française à Ankara. Ils remercient également les UMR 8591, 6266, 1572, 8586 et l'Université Paris Diderot-Paris 7 qui ont participé au soutien financier de ce programme.

\section{RÉFÉRENCES BIBLIOGRAPHIQUES}

AYDAR E., GOURGAUD A., ULUSOY I., DIGONNET F., LABAZUY P., SEN E., BAYHAN H., KERTTAS T., 1 TOLLUOGLU U., 2003 - Morphological analysis of active Mount Nemrut stratovolcano, eastern Turkey: evidences and possible impact areas of future eruption. Journal of Volcanology and Geothermal Research, 123, 301-312.

BAR-MATTHEWS M., AYALON A., \& KAUFMAN A., 1997 - Late Quaternary paleoclimate in the Eastern Mediterranean region from stable isotope analysis of spelethems at Soreq Cave, Israel. Quaternary Research, 47, 155-168.

BARTOV Y., STEIN M., ENZEL Y., AGNON A., \& RECHES Z., 2002 - Lake levels and sequence stratigraphy of Lake Lisan, the late pleistocene of the Dead Sea. Quaternary Research, 57, 9-21.

CHRISTOL A., 2005 - Mise en évidence et problématique des variations de niveaux du lac de Van pendant le Pléistocène et l'Holocène. Mémoire de DEA, Université Paris 7, 71 p. (inédit).

CHRISTOL A., KUZUCUOGLU C., FORT M., KARAYBIYIKOGLU M., MOURALIS D., BRUNSTEIN D., DOGU A-F., AKKÖPRÜ E., FONTUGNE M., \& ZORER H., 2008a Apports de l'étude Géomorphologique des terrasses fluvio-lacustres du lac de Van (Turquie) à la connaissance des paléoenvironnements en Anatolie orientale. In D. Galop (ed.), Paysages et Environnements. De la reconstitution du passé aux modèles prospectifs. Annales littéraires, Série «Environnement, sociétés et archéologie», Presse Universitaire de Franche-Comté, Besançon, 115-130.

CHRISTOL A., KUZUCUOGLU C., FORT M., DOGU A.-F., AKKÖPRÜ E., BRUNSTEIN D., MOURALIS D., FONTUGNE M., ZORER H., REYSS J.-L., 2008b - Morphogenesis, sedimentary records and palaeogeography of the Holocene and Pleistocene Van lake terraces (Turkey). Geophysical Research Abstracts, vol. 10, EGU (Vienna) 1607-7962/gra/EGU2008-A-12377.

ÇUBUKÇU E., 2008 - Evolution pétrologique du strato-volcan Nemrut Dagi (Turquie): magmatisme hyperalcalin en domaine de collision. Thèse de Doctorat, Université Blaise Pascal, Clermont-Ferrand II, $221 \mathrm{p}$.

DEGENS E.T., \& KURTMANN F., 1978 - Geology of Lake Van. MTA Press 169, Ankara.

DJAMALI M., BEAULIEU de J.-L., SHAH-HOSSEINI M., ANDRIEU-PONEL V., PONEL P., AMINI A., AKHANI H. LEROY S., STEVENS L., LAHIJANI H., \& BREWER S., 2008 - A late Pleistocene long pollen record from Lake Urmia, NW Iran. Quaternary Research, 69, 413-420.

FONTUGNE M., KUZUCUOGLU C., KARABIYIKOGLU M. HATTÉ C., \& PASTRE J-F., 1999 - From Pleniglacial to Holocene. A ${ }^{14} \mathrm{C}$ chronostratigraphy of environmental changes in the Konya Plain, Turkey. Quaternary Science Reviews, 18 (4-5), 573-592.

HAZAN N., STEIN M., AGNON A., MARCO S., NADEL D., NEGENDANK J.F.W., SCHWAB M.J., \& NEEV D., 2005 The late Quaternary limnological history of lake Kinneret (Sea of Galilee), Israel. Quaternary Research, 63, 60-77.

HEUMANN G., LITT T., KRASTEL S., STURM M., \& ÖRÇEN S., 2008 - Palynological investigations based on lacustrine sediments from Lake Van, Turkey (new ICDP site). Geophysical Research Abstracts, vol. 10, EGU- 08632, Vienna.

JUNG D., KELLER J., \& ECKHARDT F.-J., 1978 - Heavy mineral contents and geochemistry of pumice glass from tephra layers in sediments of Lake Van (East Anatolia). In E. Degens \& F. Kurtmann (eds), Geology of Lake Van. MTA Press 169, Ankara, 102-110.

KADIOGLU M., SEN Z., \& BATUR E., 1997 - The greatest sodawater lake in the world and how it is influenced by climatic change. Annals Geophysicae, 15, 1489-1497.

KEMPE S., LANDMANN G., \& MÜLLER G., 2002 - A floating varve chronology from the last glacial maximum terrace of Lake Van/ Turkey. Zeithschrift Geomorphologie, N.F., Suppl. Bd. 126, 97-114.

KIPFER R., AESCHBACH-HERTIG W., BAUR H., HOFER M., IMBODEN D.M., \& SIGNER P., 1994 - Injection of mantle type helium into Lake Van (Turkey): the clue for quantifying deep water renewal. Earth andPlanetary Science Letters, 125, 357-370. 
KUZUCUOGLU C., \& ROBERTS N., 1998 - Evolution de l'environnement en Anatolie de 20000 à 6000 BP. Paléorient, 23 (2), 7-24.

KUZUCUOGLU C., BERTAUX J., BLACK S., DENEFLE M., FONTUGNE M., KARABIYIKO LU M., KASHIMA K., LIMONDIN-LOZOUET N., MOURALIS D., \& ORTH P., 1999 Reconstruction of climatic changes during the Late Pleistocene, based on sediment records from the Konya Basin (Central Anatolia, Turkey). Geology Journal, Special Issue on Turkish Geology, 34, 175-198.

KUZUCUOGLU C., KARABIYIKOGLU M., DOGU A-F., CHRISTOL A., FORT M., AKKÖPRÜ E., MOURALIS D., BRUNSTEIN D., ZORER H., \& FONTUGNE M., 2007 - Past high magnitude stands of Van lake: geomorphological and hydrological implications. A contribution to identifying climate forcing, recent tectonic impacts and relationships to volcanic activity during Upper Pleistocene. Symposium "Quaternary of Turkey", TURQUA, Istanbul.

KUZUCUOGLU C., MOURALIS D., CHRISTOL, A., AKKOPRU, E., DOGU A.-F., FORT M., BRUNSTEIN D., FONTUGNE M., GUILLOU H., SCAILLET S., ZORER H., REYSS J.-L., LAMOTHE M., KIYAK $\mathbf{N}_{\text {, GAUTHIER }} \mathrm{A}_{\text {, \& GUICHARD }}$ F., 2008 - The «ANOVAN» Project: Palaeoenvironments, palaeogeography, volcanic events and human societies in the Van lake basin - eastern Turkey - during Upper Pleistocene and Holocene. Geophysical Research Abstracts, vol. 10, EGU (Vienna) 1607-7962 gra/EGU2008-A-12378.

KUZUCUOGLU C., CHRISTOL A.-M., MOURALIS D., DOGU A-F., AKKOPRU E., FORT M., BRUNSTEIN D., ZORER H., FONTUGNE M., KARABIYIKOGLU M., SCAILLET S., REYSS J.-L., \& GUILLOU H., 2010 - Upper Pleistocene terraces of Van lake (Turkey). Journal of Quaternary Sciences, 25 (7), 1124-1137.

LANDMANN G., REIMER A., LEMCKE G., \& KEMPE S., 1996 - Dating late glacial abrupt climate changes in the 14570 years long continuous varve record of Lake Van, Turkey. Paleogeography, Paleoclimatology, Paleoecology, 122, 105-118.

LEMCKE G., \& STURM M., 1997 - Delta ${ }^{18} \mathrm{O}$ and trace element measurements as proxy for the reconstruction of climate changes at Lake Van (Turkey). Preliminary results. In H.N.Dalfes, G. Kukla \& $\mathrm{H}$. Weiss (eds), Third millennium BC climate change and Old World Collapse. NATO ASI Series I, 653-678.

LITT T., KRASTEL S., STURM M., DALFES N., ÖRCEN S., HEUMANN G., DEMIREL-SCHLÜTER F., \& NIESSEN F., 2008 - Lake Van Drilling Project, Turkey - a new ICDP initiative. Geophysical Research Abstracts, vol. 8, EGU-04433, Vienna.

MOURALIS D., GUILLOU H., SCAILLET S., KUZUCUOGLU C., CHRISTOL A., AKKÖPRÜ E., FONTUGNE M., DOGU A.F., ZORER H., \& KARABIYIYKOGLU M., 2007 - Les tephras interstratifiés dans les terrasses lacustres du lac de Van (Turquie) marqueurs chronostratigraphiques et identification des volcanssources (Nemrut, Süphan, etc.). Résultats préliminaires. Colloque CNRS ECLIPSE II, Paris.
MOURALIS D., KUZUCUOGLU C., AKKÖPRU E., DOGU A-F., SCAILLET S., CHRISTOL A., ZORER H., BRUNSTEIN D., FORT M., \& GUILLOU H., 2010 - Les pyroclastites du sud-ouest du lac de Van (Anatolie orientale, Turquie): implications dans la paléo-hydrographie régionale. Quaternaire, 21 (4), 425-442.

NEMEC W., \& STEEL R.J. (eds.), 1988 - Fan deltas : Sedimentology and tectonic settings. Blackie, London, $444 \mathrm{p}$.

READING H.G. (ed.), 1996 - Sedimentary environments : Processes, facies and stratigraphy. $3^{\text {rd }}$ edition Blackwell Science Ltd, $704 \mathrm{p}$.

ROBINSON S.A., BLACK S., SELLWOOD B.W., \& VALDES P.J., 2006 - A review of palaeoclimates and palaeoenvironments in the Levant and Eastern Mediterranean from 25,000 to 5000 years BP: setting the environmental background for the evolution of human civilisation. Quaternary Science Reviews, 25, 1517-1541.

SENGÖRA.M.C., \& YILMAZY., 1981 - Tethyan evolution of Turkey: A plate tectonic approach. Tectonophysics, 75 (3-4), 181-241.

SENGÖR A.M.C., ÖZEREN M.S., KESKIN M., SAKINÇ M., ÖZBAKIR A.D., \& KAYAN I., 2008 - Eastern Turkish high plateau as a small Turkic-type orogen: Implications of post-collisional crustforming processes in Turkic-type orogen. Earth Science Reviews, 90 (1-2), 1-48.

SCHWEIZER G., 1975 - Untersuchungen zur Physiogeographie von Ostanatolien und Nordwestiran, geomorphologische, klima- und hydrogeographische Studien im Vansee- und Rezaiyehsee-Gebiet. Tübinger Geographische Studien, 60, $145 \mathrm{p}$.

STUIVER M., \& REIMER P., 1993 - Extended ${ }^{14} \mathrm{C}$ data base and revised Calib $3.0{ }^{14} \mathrm{C}$ age calibration programme. Radiocarbon, $\mathbf{3 5}$, 215-230.

VALETON I., 1978 - A morphological and petrological study of the terraces around Lake Van, Turkey. In E. Degens \& F. Kurtmann (eds), Geology of Lake Van. MTA Press 169, Ankara, 64-80.

WICK A., LEMCKE G., \& STURM M., 2003 - Evidence of Lateglacial and Holocene climate change and human impact in eastern Anatolia : high resolution pollen, charcoal, isotopic and geochemical records from the laminates sediments of Lake Van. The Holocene, $13(5), 665-675$

YILMAZ Y., GÜNER Y., \& SAROGLU F., 1998 - Geology of the quaternary volcanic centres of the east Anatolia. Journal of volcanology and geothermal research, 85, 173-210.

Van ZEIST W., \& WOLDRING H., 1978 - A postglacial pollen diagram from Lake Van in the East Anatolia. Review of Palaeobotany and Palynology, 26, 249-27. 